ORNL/TM-1999/158

\title{
A MICROMECHANICAL CONSTITUTIVE MODEL OF PROGRESSIVE CRUSHING IN RANDOM CARBON FIBER POLYMER MATRIX COMPOSITES
}

H. K. Lee

S. Simunovic 
This report has been reproduced directly from the best available copy.

Available to DOE and DOE contractors from the Office of Scientific and Technical Information, P.O. Box 62, Oak Ridge, TN 37831; prices available from (615) 576-8401.

Available to the public from the National Technical Information Service, U.S. Department of Commerce, 5285 Port Royal Rd., Springfield, VA 22161.

This report was prepared as an account of work sponsored by an agency of the United States Government. Neither the United States nor any agency thereof, nor any of their employees, makes any warranty, express or implied, or assumes any legal liability or responsibility for the accuracy, completeness, or usefulness of any information, apparatus, product, or process disclosed, or represents that its use would not infringe privately owned rights. Reference herein to any specific commercial product, process, or service by trade name, trademark, manufacturer, or otherwise, does not necessarily constitute or imply its endorsement, recommendation, or favoring by the United States Government or any agency thereof. The views and opinions of authors expressed herein do not necessarily state or reflect those of the United States Government or any agency thereof. 
ORNL/TM-1999/158

\title{
A MICROMECHANICAL CONSTITUTIVE DAMAGE MODEL OF PROGRESSIVE CRUSHING IN RANDOM CARBON FIBER POLYMER MATRIX COMPOSITES
}

\author{
Computer Science and Mathematics Division \\ Haeng-Ki Lee \\ Srdan Simunovic
}

Date Published: September 1999

\author{
Prepared by the \\ OAK RIDGE NATIONAL LABORATORY \\ Oak Ridge, Tennessee 37831 \\ managed by \\ LOCKHEED MARTIN ENERGY RESEARCH CORP. \\ for the \\ U.S. DEPARTMENT OF ENERGY \\ under contract DE-AC05-96OR22464
}




\section{CONTENTS}

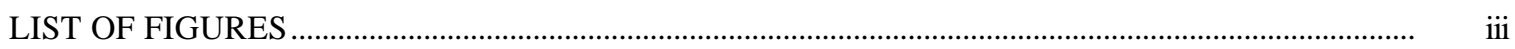

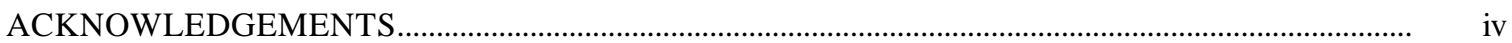

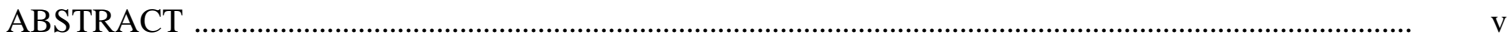

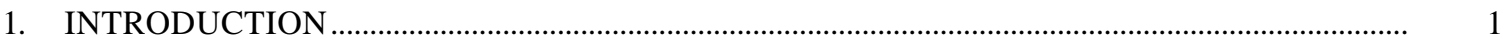

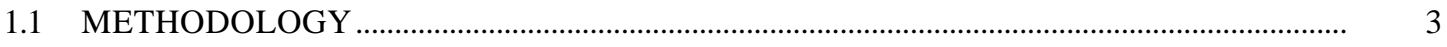

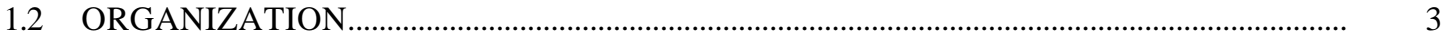

2. OVERALL ELASTOPLASTIC BEHAVIOR OF RFPCS:

A MICROMRCHANICAL FRAMEWORK …………………………………………................... 5

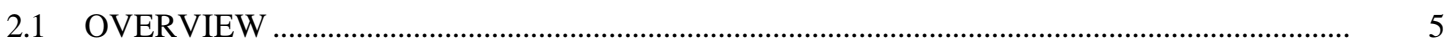

2.2 EFFECTIVE ELASTOPLASTIC BEHAVIOR OF COMPOSITES

WITH ALIGNED DISCONTINUOUS FIBERS.............................................................................

2.3 EFFECTIVE ELASTOPLASTIC BEHAVIOR OF COMPOSITES WITH RANDOMLY ORIENTED FIBERS.................................................................................... 19

2.4 ELASTOPLASTIC STRESS-STRAIN RELATIONS OF RFPCS ............................................... 25

2.4.1 Uniaxial Elastoplastic Stress-strain Relation …………………………………………..... 25

2.4.2 Biaxial Elastoplastic Stress-strain Relation......................................................................... 27

2.4.1 Triaxial Elastoplastic Stress-strain Relation .................................................................. 28

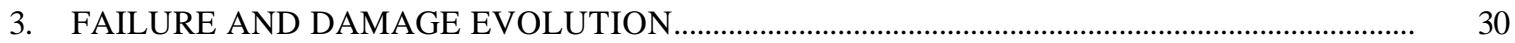

$3.1 \quad$ FAILURE IN DISCONTINUOUS FIBER COMPOSITES ......................................................... 30

3.2 EVOLUTIONARY INTERFACIAL DEBONDING:

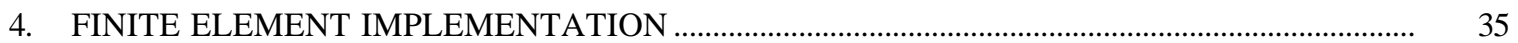

4.1 COMPUTATIONAL INTEGRATION ALGORITHM S ……………………………………….... 35

4.2 CONTINUUM TANGENT MODULI ......................................................................................... 37

5. NUMERICAL SIMULATIONS AND EXPERIMENTAL COMPARISON ………………………..... 41

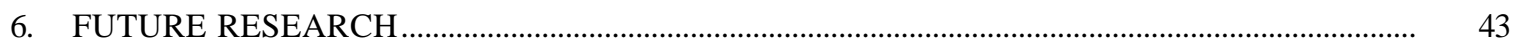

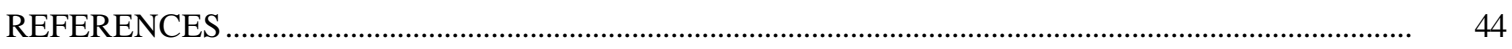

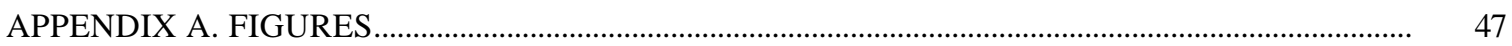




\section{LIST OF FIGURES}

Figure

Page

1 An RVE for a composite and superposition involving homogeneous strain and perturbed strain

2 Schematic description of the imaginary ellipsoid and its outward unit normal vector for an ellipsoidal inclusion...

3 Effect of the shape of fibers on the overall uniaxial elastoplastic behavior of random carbon fiber polymer matrix composites

4 Effect of the initial volume fraction of fibers on the overall uniaxial elastoplastic behavior of random carbon fiber polymer matrix composites

5 The predicted evolution of debonded fiber volume fraction corresponding to Figure 4

6 The comparison between the present prediction and experimental data (Meraghni and Benzeggagh, 1995) for overall uniaxial tensile responses of randomly oriented discontinuous fiber composites with initial fiber volume fraction of 0.5

7 The predicted evolution of debonded fiber volume fraction versus strain corresponding to Figure 6 


\section{ACKNOWLEDGEMENTS}

This research was sponsored by the U.S. Department of Energy, Assistant Secretary for

Energy Efficiency and Renewable Energy, Office of Transportation Technologies, Lightweight Materials Program, under contract DE-AC05-96OR22464 with Lockheed Martin Energy Research Corporation. The research was supported in part by an appointment to the Oak Ridge National Laboratory Postdoctoral Research Associates Program administered jointly by the Oak Ridge National Laboratory and the Oak Ridge Institute for Science and Education. 


\begin{abstract}
A micromechanical damage constitutive model is presented to predict the overall elastoplastic behavior and damage evolution in random carbon fiber polymer matrix composites (RFPCs). To estimate the overall elastoplastic damage responses, an effective yield criterion is derived based on the ensemble-volume averaging process and first-order effects of eigenstrains due to the existence of spheroidal (prolate) fibers. The proposed effective yield criterion, together with the assumed overall associative plastic flow rule and hardening law, constitutes the analytical foundation for the estimation of effective elastoplastic behavior of ductile matrix composites. First, an effective elastoplastic constitutive damage model for aligned fiber-reinforced composites is proposed. A micromechanical damage constitutive model for RFPCs is then developed. The average process over all orientations upon governing constitutive field equations and overall yield function for aligned fiber-reinforced composites is performed to obtain the constitutive relations and effective yield function of RFPCs. The discrete numerical integration algorithms and the continuum tangent operator are also presented to implement the proposed damage constitutive model. The damage constitutive model forms the basis for the progressive crushing in composite structures under impact loading.
\end{abstract}




\section{INTRODUCTION}

The goal to provide lighter-weight, more fuel-efficient automobiles capable of greater crashworthiness has provided an incentive for the continued development of advanced materials. Carbon fiber composites, which are a new breed of high-strength materials, have attracted worldwide attention and hold great promise, but are significantly more brittle in general, when compared with other polymer composites. Thus, they are used in composites with a lightweight matrix, such as an epoxy resin (Donnet and Bansal, 1990). It is well known that organic matrix, fiber-reinforced composites are very susceptible to impact damage, especially at low velocities. Low-velocity impact can cause significant damage (i.e., matrix cracks and delaminations) inside the composites. Such damage is very difficult to detect and may cause a significant reduction in the strength and stiffness of the materials.

Damage accumulation in fiber-reinforced organic matrix composites is a complicated, progressive phenomenon (Groves et al., 1987; Meraghni and Benzeggagh, 1995; Meraghni et al., 1996). It involves multiple failure modes such as matrix cracking, fiber breakage, delamination, etc. Any of these failure modes may begin in an early loading stage and progressively accumulate inside the materials (Wang, 1984; Caslini et al., 1987). The presence of damage can affect the mechanical properties, and subsequent response of composites. Accordingly, it is essential in structural application of the composites that the accumulated damage can be predicted, and the effect of such damage on the response and failure of the structures can be determined accurately. Analyses and tests to assess the crushing of composites during low-velocity impact have been carried out (Keal, 1983; Price and Hull, 1987). Experimental investigations on fiber-reinforced tubes and cones indicate a wide range of material damage, such as matrix crushing, delamination, and fiber breakage. Matrix cracking and delamination often show a rather slowly progressing failure with high energy dissipation, while fiber breakage may initiate catastrophic collapse of the entire structure with little dissipation of kinetic energy. It has been noted from these previous studies that the microscopic failure behavior of the fiber-reinforced composites is still not completely understood. Nevertheless, several attempts to simulate composite crash damage have been made (Murray, 1989; Matzenmiller and Schweizerhof, 1991; Simunovic and Zacharia, 1996). A more detailed failure review of fiber-reinforced composites can be found in Matzenmiller and Schweizerhof (1991), Kutlu and Chang (1995), and Meraghi and Benzeggagh (1995, 1996).

In general, the field of traditional continuum mechanics is based on the continuity, isotropy, and homogeneity of materials. Therefore, it cannot directly solve the problem for heterogeneous composites, since fibers or particles are present within the composites and have a significant effect on the mechanical properties of materials. Hence, micromechanics has been developed to solve the problem on a finer scale and 
encompasses mechanics related to microstructures of materials. Although the concept of micromechanics can be traced back to the late 1930s (e.g., Goodier, 1937; Eshelby, 1957, 1959, 1961), micromechanics has only widely been developed since 1980s (e.g., Mura, 1987; Nemat-Nasser and Hori, 1993; Mura et al., 1996).

Micromechanical approaches enable us to evaluate and predict local stress and strain fields in each constituent. Hence, the derivation of the constitutive equations in the form of a phenomenological parameter model from entirely micromechanical considerations is required to perform the rigorous analysis of composite structures. Such an approach is more justified in the case of composite materials reinforced with randomly oriented discontinuous fibers. Indeed, the microstructure of these materials, the complexity of damage mechanisms, and the diversity of their scenarios significantly influence their overall properties. Furthermore, because of the natural tendency of the structure to acquire lower energy modes, both material and structural damage processes need to be thoroughly understood and modeled to simulate and eventually design the desirable sustained crush of the component. Therefore, accurate analysis and the ability to simulate the complete response of components and systems of RFPCs are essential and require accurate micromechanical damage constitutive models.

Choi and Chang (1992) developed a model for predicting damage in graphite/epoxy laminated composites resulting from low-velocity point impact. A transient dynamic finite element analysis was adopted for calculating the stresses and strains inside the composites during impact. In their derivation, failure criteria were proposed to predict the initial matrix cracking and the size of the interface delaminations in the composites. On the other hand, a micromechanical analysis based on the modified Mori-Tanaka method was performed by Meraghi and Benzeggagh $(1995,1996)$ to address the effect of matrix degradation and interfacial debonding on stiffness reduction in a random discontinuous-fiber composite. Their modeling relied on an experimental approach, developed through a methodology of experimental identification of basic damage mechanisms, which involved amplitude analysis of acoustic emission and microscopic observations. Tohgo and Weng (1994) and Zhao and Weng (1995, 1996, 1997) proposed progressive interfacial damage models for ductile matrix composites. They used Weibull's (1951) probability distribution function to describe the probability of particle debonding. Recently, Ju and Lee (1999) developed a micromechanical damage model to predict the overall elastoplastic behavior and damage evolution in ductile matrix composites. In their derivation, to estimate the overall elastoplastic-damage behavior, an effective yield criterion was derived based on the ensemble-volume averaging procedure and the first-order effects of eigenstrains stemming from the existence of inclusions. 


\subsection{METHODOLOGY}

Following the work of Zhao and Weng (1995) and Ju and Lee (1999), we propose three dimensional micromechanical damage constitutive models to assess effective elastoplastic behavior of damaged composite materials and address the damage response of RFPCs. The damage constitutive model forms the basis for assessing the progressive crushing in composite structures under impact loading. In our derivation, fibers are assumed to be elastic spheroids that are randomly dispersed in a ductile polymer matrix. Furthermore, the ductile matrix behaves elastoplastically under arbitrary three-dimensional loading/unloading. All fibers are assumed to be non-interacting for dilute composite medium and initially embedded firmly in the matrix with perfect interfaces. After the interfacial debonding between fibers and the matrix, these partially debonded fibers are regarded as equivalent, transversely isotropic inclusions. It should be noted that the scope of this work is to predict the overall damage behavior of RFPCs globally; therefore, the local microcrack propagation and void nucleation at the interfaces are ignored in our derivation. However, it is acceptible to extend the proposed damage model to accommodate local damage evolution, once new damage growth model and failure criterion are developed based on rigorous experiments. In the future, the present damage constitutive model will be extended and be able to account for the effect of interactions of the composite with high fiber volume fraction. In addition, a new failure criterion based on experimental verifications of randomly oriented, discontinuous fiber-reinforced composites will also be proposed to perform failure analysis for RFPCs. Finally, the present micromechanical damage constitutive models will be implemented into the finite element code, DYNA-3D, to address the progressive crushing in composite structures under impact loading.

\subsection{ORGANIZATION}

This report is organized as follows. In Section 2, to predict the overall elastoplastic-damage behavior of RFPCs, an "effective yield criterion" is micromechanically constructed based on the ensemble-volume averaging procedure and the first-order effects of eigenstrains due to the existence of discontinuous, randomly oriented fibers. The proposed elastoplastic-damage formulation is applied to uniaxial, biaxial, and triaxial loading conditions. An evolutionary, interfacial debonding model is considered in accordance with the Weibull's statistical function in Section 3. The explicit relationship is also derived in Section 3 to relate the average internal stress inside a fiber and the macroscopic total strain. Computational aspects of the proposed elastoplastic-damage model are the main concern in Section 4. The discrete numerical integration algorithm is employed to integrate the rate equations in the effective elastoplastic model. The continuum tangent

operator, based on the continuous rate equations, is derived for a special case of implicit time integration 
algorithms in nonlinear finite element methods. In Section 5, the present predictions are compared with other theoretical predictions and the experimental data. Finally, future research is summarized in Section 6. 


\section{OVERALL ELASTOPLASTIC BEHAVIOR OF RFPCs: \\ A MICROMECHANICAL FRAMEWORK}

\subsection{OVERVIEW}

The prediction and estimation of the overall "effective" mechanical properties of random heterogeneous multiphase materials are of great interest to researchers and engineers in many science and engineering disciplines. The so-called "effective" properties of a heterogeneous composite are obtained by volume- and ensemble-averaging processes over a representative volume element (RVE) featuring a "mesoscopic" length scale, which is much larger than the characteristic length scale of inclusions (inhomogeneities), but smaller than the characteristic length scale of a macroscopic specimen. In this report, all inclusions are assumed to be embedded firmly in the matrix with perfect interfaces initially. Furthermore, we assume that statistical homogeneity holds. Therefore, effective (averaged) material properties remain the same for arbitrary averaging domains inside a composite medium. As a result, heterogeneous composites can be represented by equivalent homogeneous continuum media with appropriately defined effective properties. Mathematically, this procedure is related to the homogenization method. Examples of heterogeneous inclusions composites are abundant (e.g., graphite/epoxy composites, ceramic matrix composites, porous and cracked media, polymer-blended soils, rocks, etc.).

To obtain "effective" constitutive equations and properties of random heterogeneous composites, one typically performs the ensemble-volume averaging process (homogenization) within an RVE. To avoid the truncation errors of Green's functions outside the domain of an RVE, an ellipsoidal RVE itself is embedded in an infinite (and identical) matrix material within our framework. The entire assembly is subjected to specified far-field stresses or strains. The volume-averaged stress tensor is defined as

$$
\overline{\boldsymbol{\sigma}} \equiv \frac{1}{V} \int \boldsymbol{\sigma}(\mathbf{x}) d \mathbf{x}=\frac{1}{V}\left[\int_{V_{m}} \boldsymbol{\sigma}(\mathbf{x}) d \mathbf{x}+\sum_{r=1}^{n} \int_{V_{r}} \boldsymbol{\sigma}(\mathbf{x}) d \mathbf{x}\right]
$$

where $V$ is the volume of an RVE, $V_{m}$ is the volume of the matrix, $V_{r}$ is the volume of the $r$ th-phase inhomogeneities, and $n$ denotes the number of inclusions of different material properties (excluding the matrix). Similarly, the volume-averaged strain tensor is defined as

$$
\overline{\boldsymbol{\epsilon}} \equiv \frac{1}{V} \int_{V} \boldsymbol{\epsilon}(\mathbf{x}) d \mathbf{x}=\frac{1}{V}\left[\int_{V_{m}} \boldsymbol{\epsilon}(\mathbf{x}) d \mathbf{x}+\sum_{r=1}^{n} \int_{V_{r}} \boldsymbol{\epsilon}(\mathbf{x}) d \mathbf{x}\right] \equiv \frac{1}{V}\left[V_{m} \overline{\boldsymbol{\epsilon}}_{m}+\sum_{r=1}^{n} V_{r} \overline{\boldsymbol{\epsilon}}_{r}\right]
$$

Moreover, the effective elastic stiffness tensor $\mathbf{C}_{*}$ of the composite is defined through

$$
\overline{\boldsymbol{\sigma}} \equiv \mathbf{C}_{*}: \overline{\boldsymbol{\epsilon}}
$$


where ":" signifies the tensor contraction.

According to Eshelby's equivalence principle (Eshelby, 1957, 1961), the perturbed strain field $\boldsymbol{\epsilon}^{\prime}(\mathbf{x})$ induced by inhomogeneities (inclusions with properties different from those of the homogeneous matrix) can be related to specified eigenstrain $\boldsymbol{\epsilon}^{*}(\mathbf{x})$ by replacing the inhomogeneities with the matrix material. That is, for the domain of $q$-phase inhomogeneities with the elasticity tensor $\mathbf{C}_{q}$, we have

$$
\mathbf{C}_{q}:\left[\boldsymbol{\epsilon}^{o}+\boldsymbol{\epsilon}^{\prime}(\mathbf{x})\right]=\mathbf{C}_{0}:\left[\boldsymbol{\epsilon}^{o}+\boldsymbol{\epsilon}^{\prime}(\mathbf{x})-\boldsymbol{\epsilon}^{*}(\mathbf{x})\right]
$$

where $\mathbf{C}_{0}$ is the stiffness tensor of the matrix and $\boldsymbol{\epsilon}^{o}$ is the uniform strain field by far-field loads for a homogeneous matrix material only. $\mathbf{C}_{0}$ and $\mathbf{C}_{q}$ could be isotropic or anisotropic if the eigenstrain field $\boldsymbol{\epsilon}^{*}(\mathbf{x})$ is uniform in $V$. In Figure 1, the strain at any point within an RVE is decomposed into two parts: (a) the uniform strain $\boldsymbol{\epsilon}^{o}$ (without inhomogeneities), and (b) the perturbed strain $\boldsymbol{\epsilon}^{\prime}(\mathbf{x})$ due to distributed eigenstrains $\boldsymbol{\epsilon}^{*}(\mathbf{x})$. It is emphasized that the eigenstrain $\boldsymbol{\epsilon}^{*}(\mathbf{x})$ is nonzero in the inclusion domain and zero in the matrix domain, respectively. In particular, the perturbed strain field induced by distributed eigenstrains $\boldsymbol{\epsilon}^{*}$ can be expressed as

$$
\boldsymbol{\epsilon}^{\prime}(\mathbf{x})=\int_{V} \mathbf{G}\left(\mathbf{x}-\mathbf{x}^{\prime}\right): \boldsymbol{\epsilon}^{*}\left(\mathbf{x}^{\prime}\right) d \mathbf{x}^{\prime}
$$

where $V$ is the volume of an RVE and $\mathbf{x}, \mathbf{x}^{\prime} \in V$. In addition, $\mathbf{G}$ is the second derivative of the Green's function in a linear elastic homogeneous matrix. For example, for a linear, elastic isotropic matrix, we have

$$
\begin{aligned}
G_{i j k l}= & \frac{1}{8 \pi\left(1-\nu_{0}\right) r^{3}}\left[-15 n_{i} n_{j} n_{k} n_{l}+3 \nu_{0}\left(\delta_{i k} n_{j} n_{l}+\delta_{i l} n_{j} n_{k}+\delta_{j k} n_{i} n_{l}+\delta_{j l} n_{i} n_{k}\right)\right. \\
& \left.+3 \delta_{i j} n_{k} n_{l}+3\left(1-2 \nu_{0}\right) \delta_{k l} n_{i} n_{j}-\left(1-2 \nu_{0}\right) \delta_{i j} \delta_{k l}+\left(1-2 \nu_{0}\right)\left(\delta_{i k} \delta_{j l}+\delta_{i l} \delta_{j k}\right)\right]
\end{aligned}
$$

where $\mathbf{r}=\mathbf{x}-\mathbf{x}^{\prime}, r=\left\|\mathbf{x}-\mathbf{x}^{\prime}\right\|$ and $\mathbf{n}=\mathbf{r} / r$. Further, summation convention applieds, $\delta_{i j}$ denotes the Kronecker delta and $\nu_{0}$ is Poisson's ratio of the homogeneous matrix. Eshelby (1957, 1959) used a fourthrank tensor $\mathbf{S}$, which is traditionally called Eshelby's tensor, to describe the strain and stress fields in the inclusion domain. The Eshelby's tensor is defined as

$$
\mathbf{S}(\mathbf{x})=\int_{\Omega} \mathbf{G}\left(\mathbf{x}-\mathbf{x}^{\prime}\right) d \mathbf{x}^{\prime}
$$

in which $\mathbf{x}$ is the local point inside the inclusion domain $\Omega$.

Total strain at any point $\mathbf{x}$ in the matrix is given by superposition of uniform strain $\boldsymbol{\epsilon}^{o}$ and the perturbed strain $\boldsymbol{\epsilon}^{\prime}$ induced by inclusions (inhomogeneities)

$$
\boldsymbol{\epsilon}(\mathbf{x})=\boldsymbol{\epsilon}^{o}+\boldsymbol{\epsilon}^{\prime}(\mathbf{x})=\boldsymbol{\epsilon}^{o}+\int_{V} \mathbf{G}\left(\mathbf{x}-\mathbf{x}^{\prime}\right): \boldsymbol{\epsilon}^{*}\left(\mathbf{x}^{\prime}\right) d \mathbf{x}^{\prime}
$$


Therefore, the volume-averaged strain tensor is given by

$$
\overline{\boldsymbol{\epsilon}}=\boldsymbol{\epsilon}^{o}+\frac{1}{V} \int_{V} \int_{V} \mathbf{G}\left(\mathbf{x}-\mathbf{x}^{\prime}\right): \boldsymbol{\epsilon}^{*}\left(\mathbf{x}^{\prime}\right) d \mathbf{x}^{\prime} d \mathbf{x}=\boldsymbol{\epsilon}^{o}+\frac{1}{V} \int_{V}\left[\int_{V} \mathbf{G}\left(\mathbf{x}-\mathbf{x}^{\prime}\right) d \mathbf{x}\right]: \boldsymbol{\epsilon}^{*}\left(\mathbf{x}^{\prime}\right) d \mathbf{x}^{\prime}
$$

When considering the strain and stress fields at a local point $\mathbf{x}$ that is outside inclusion, we define a fourth-rank tensor $\overline{\mathbf{G}}(\mathbf{x})$, which is called the exterior-point Eshelby's tensor as (see Eshelby, 1959; Mura, 1987)

$$
\overline{\mathbf{G}}(\mathbf{x}) \equiv \int_{\Omega} \mathbf{G}\left(\mathbf{x}-\mathbf{x}^{\prime}\right) d \mathbf{x}^{\prime}
$$

where $\mathbf{x} \in V-\Omega$.

Exterior-point Eshelby's tensor $\mathbf{G}(\mathbf{x})$ of an ellipsoidal inclusion can be derived by introducing an outward unit normal vector $\hat{\mathbf{n}}$ at a matrix point $\mathbf{x}$ on the new imaginary ellipsoid surface (Figure 2) which can be defind as

$$
\hat{n}_{i}=\frac{x_{i}}{\left(a_{I}^{2}+\vartheta\right) \sqrt{\Theta(\vartheta)}}
$$

where

$$
\Theta(\vartheta) \equiv \Theta_{i}(\vartheta) \Theta_{i}(\vartheta)
$$

and

$$
\Theta_{i}(\vartheta) \equiv \frac{x_{i}}{a_{I}^{2}+\vartheta}
$$

in which $a_{I}(I=1,2,3)$ is one of the three semi-axes of the ellipsoid, and $\vartheta$ is taken as positive and can be uniquely solved in terms of local point $\mathbf{x}$ of matrix and $a_{I}$. With the help of the above definitions, the exterior-point Eshelby's tensor $\overline{\mathbf{G}}(\mathbf{x})$ can be explicitly expressed as (see, Ju and Sun, 1999)

$$
\begin{aligned}
\overline{\mathbf{G}}(\mathbf{x})= & \mathcal{S}_{I K}^{(1)}(\vartheta) \delta_{i j} \delta_{k l}+\mathcal{S}_{I J}^{(2)}(\vartheta)\left(\delta_{i k} \delta_{j l}+\delta_{i l} \delta_{j k}\right)+\mathcal{S}_{I}^{(3)}(\vartheta) \delta_{i j} \hat{n}_{k} \hat{n}_{l} \\
& +\mathcal{S}_{K}^{(4)}(\vartheta) \delta_{k l} \hat{n}_{i} \hat{n}_{j}+\mathcal{S}_{I}^{(5)}(\vartheta)\left(\delta_{i k} \hat{n}_{j} \hat{n}_{l}+\delta_{i l} \hat{n}_{j} \hat{n}_{k}\right) \\
& +\mathcal{S}_{J}^{(6)}(\vartheta)\left(\delta_{j k} \hat{n}_{i} \hat{n}_{l}+\delta_{j l} \hat{n}_{i} \hat{n}_{k}\right)+\mathcal{S}_{I J K L}^{(7)}(\vartheta) \hat{n}_{i} \hat{n}_{j} \hat{n}_{k} \hat{n}_{l}
\end{aligned}
$$

As a special case, if two of the three semi-axes of the ellipsoid are the same, then the ellipsoid will become a spheroid. Let us assume that $a_{1} \neq a_{2}=a_{3}$, where the spheroid aspect ratio $\alpha$ is defined as $\alpha \equiv a_{1} / a_{2}$. Following Ju and Sun (1999), if all fibers are spheroid and the matrix is linear elastic, then the components of the exterior-point Eshelby's tensor can be written as

$$
\begin{aligned}
& \mathcal{S}_{11}^{(1)}(\vartheta)=\left[-4 \nu_{0}-\frac{2}{\alpha^{2}-1}\right] g(\vartheta)-\frac{2}{3\left(\alpha^{2}-1\right)} \rho_{1}^{3}(\vartheta)+\left[4 \nu_{0}+\frac{2}{\alpha^{2}-1}\right] \rho_{1}(\vartheta) \rho_{2}^{2}(\vartheta) \\
& \mathcal{S}_{12}^{(1)}(\vartheta)=\mathcal{S}_{13}^{(1)}(\vartheta)=\left[-4 \nu_{0}+\frac{2 \alpha^{2}+1}{\alpha^{2}-1}\right] g(\vartheta)+\left[4 \nu_{0}-\frac{2 \alpha^{2}}{\alpha^{2}-1}\right] \rho_{1}(\vartheta) \rho_{2}^{2}(\vartheta)
\end{aligned}
$$




$$
\begin{aligned}
& \mathcal{S}_{21}^{(1)}(\vartheta)=\mathcal{S}_{31}^{(1)}(\vartheta)=\left[-2 \nu_{0}-\frac{2 \alpha^{2}+1}{\alpha^{2}-1}\right] g(\vartheta)-\frac{2 \alpha^{2}}{\alpha^{2}-1} \rho_{1}(\vartheta) \rho_{2}^{2}(\vartheta) \\
& \mathcal{S}_{22}^{(1)}(\vartheta)=\mathcal{S}_{23}^{(1)}(\vartheta)=\mathcal{S}_{32}^{(1)}(\vartheta)=\mathcal{S}_{33}^{(1)}(\vartheta)=\left[-2 \nu_{0}+\frac{4 \alpha^{2}-1}{4\left(\alpha^{2}-1\right)}\right] g(\vartheta)+\frac{\alpha^{2}}{2\left(\alpha^{2}-1\right)} \frac{\rho_{2}^{4}(\vartheta)}{\rho_{1}(\vartheta)} \\
& \mathcal{S}_{11}^{(2)}(\vartheta)=\left[-4 \nu_{0}+\frac{4 \alpha^{2}-2}{\alpha^{2}-1}\right] g(\vartheta)-\frac{2}{3\left(\alpha^{2}-1\right)} \rho_{1}^{3}(\vartheta)-\left[4 \nu_{0}-\frac{4 \alpha^{2}-2}{\alpha^{2}-1}\right] \rho_{1}(\vartheta) \rho_{2}^{2}(\vartheta) \\
& \mathcal{S}_{12}^{(2)}(\vartheta)=\mathcal{S}_{13}^{(2)}(\vartheta)=\mathcal{S}_{21}^{(2)}(\vartheta)=\mathcal{S}_{31}^{(2)}(\vartheta)=\left[-\nu_{0}-\frac{\alpha^{2}+2}{\alpha^{2}-1}\right] g(\vartheta)-\left[2 \nu_{0}+\frac{2}{\alpha^{2}-1}\right] \rho_{1}(\vartheta) \rho_{2}^{2}(\vartheta) \\
& \mathcal{S}_{22}^{(2)}(\vartheta)=\mathcal{S}_{23}^{(2)}(\vartheta)=\mathcal{S}_{32}^{(2)}(\vartheta)=\mathcal{S}_{33}^{(2)}(\vartheta)=\left[2 \nu_{0}-\frac{4 \alpha^{2}-7}{4\left(\alpha^{2}-1\right)}\right] g(\vartheta)+\frac{\alpha^{2}}{2\left(\alpha^{2}-1\right)} \frac{\rho_{2}^{4}(\vartheta)}{\rho_{1}(\vartheta)}
\end{aligned}
$$

where

$$
g(\vartheta)= \begin{cases}-\frac{\alpha^{2}}{\alpha^{2}-1} \frac{\rho_{2}^{2}(\vartheta)}{\rho_{1}(\vartheta)}+\frac{\alpha}{\left(\alpha^{2}-1\right)^{3 / 2}} \ln \left[\left(\alpha^{2}-1\right)^{1 / 2} \rho_{2}(\vartheta)+\frac{\alpha \rho_{2}(\vartheta)}{\rho_{1}(\vartheta)}\right], & \text { for } \alpha>1 \\ -\frac{\alpha^{2}}{\alpha^{2}-1} \frac{\rho_{2}^{2}(\vartheta)}{\rho_{1}(\vartheta)}+\frac{\alpha}{\left(1-\alpha^{2}\right)^{3 / 2}} \tan ^{-1} \frac{\alpha}{\left(1-\alpha^{2}\right)^{1 / 2} \rho_{1}(\vartheta)}, & \text { for } \alpha<1\end{cases}
$$

and

$$
\begin{aligned}
& \rho_{I}(\vartheta) \equiv \frac{a_{I}}{\sqrt{a_{I}^{2}+\vartheta}} \\
& \rho(\vartheta) \equiv\left[\rho_{1}(\vartheta) \rho_{2}(\vartheta) \rho_{3}(\vartheta)\right]^{1 / 3}
\end{aligned}
$$

Furthermore, interior-point Eshelby's tensor of a spheroidal inclusion can be obtained by letting $\vartheta=0$ in Equations 15-21 (dropping 0)

$$
\begin{aligned}
\mathbf{S}(\mathbf{x}) & \equiv \int_{\Omega} \mathbf{G}\left(\mathbf{x}-\mathbf{x}^{\prime}\right) d \mathbf{x}^{\prime}, \quad \mathbf{x} \in \Omega \\
& =\mathcal{S}_{I K}^{(1)} \delta_{i j} \delta_{k l}+\mathcal{S}_{I J}^{(2)}\left(\delta_{i k} \delta_{j l}+\delta_{i l} \delta_{j k}\right)
\end{aligned}
$$

with

$$
\begin{aligned}
& \mathcal{S}_{11}^{(1)}=\left[4 \nu_{0}+\frac{2}{\alpha^{2}-1}\right] \eta+4 \nu_{0}+\frac{4}{3\left(\alpha^{2}-1\right)} \\
& \mathcal{S}_{12}^{(1)}=\mathcal{S}_{13}^{(1)}=\left[4 \nu_{0}-\frac{2 \alpha^{2}+1}{\alpha^{2}-1}\right] \eta+4 \nu_{0}-\frac{2 \alpha^{2}}{\alpha^{2}-1} \\
& \mathcal{S}_{21}^{(1)}=\mathcal{S}_{31}^{(1)}=\left[-2 \nu_{0}-\frac{1+2 \alpha^{2}}{\alpha^{2}-1}\right] \eta-\frac{2 \alpha^{2}}{\alpha^{2}-1} \\
& \mathcal{S}_{22}^{(1)}=\mathcal{S}_{23}^{(1)}=\mathcal{S}_{32}^{(1)}=\mathcal{S}_{33}^{(1)}=\left[-2 \nu_{0}-\frac{4 \alpha^{2}-1}{4\left(\alpha^{2}-1\right.}\right] \eta+\frac{\alpha^{2}}{2\left(\alpha^{2}-1\right)} \\
& \mathcal{S}_{11}^{(2)}=\left[-4 \nu_{0}+\frac{4 \alpha^{2}-2}{\alpha^{2}-1}\right] \eta-4 \nu_{0}+\frac{12 \alpha^{2}-8}{3\left(\alpha^{2}-1\right)} \\
& \mathcal{S}_{12}^{(2)}=\mathcal{S}_{13}^{(2)}=\mathcal{S}_{21}^{(2)}=\mathcal{S}_{31}^{(2)}=\left[-\nu_{0}-\frac{\alpha^{2}+2}{\alpha^{2}-1}\right] \eta-2 \nu_{0}-\frac{2}{\alpha^{2}-1} \\
& \mathcal{S}_{22}^{(2)}=\mathcal{S}_{23}^{(2)}=\mathcal{S}_{32}^{(2)}=\mathcal{S}_{33}^{(2)}=\left[2 \nu_{0}-\frac{4 \alpha^{2}-7}{4\left(\alpha^{2}-1\right)}\right] \eta+\frac{\alpha^{2}}{2\left(\alpha^{2}-1\right)}
\end{aligned}
$$

where

$$
\eta=g(0)= \begin{cases}\frac{\alpha}{\left(\alpha^{2}-1\right)^{3 / 2}}\left[\cosh ^{-1} \alpha-\alpha\left(\alpha^{2}-1\right)^{1 / 2}\right], & \text { for } \alpha>1 \\ \frac{\alpha}{\left(1-\alpha^{2}\right)^{3 / 2}}\left[\alpha\left(1-\alpha^{2}\right)^{1 / 2}-\cos ^{-1} \alpha\right], & \text { for } \alpha<1\end{cases}
$$


Alternatively, Eshelby's tensor for a spheroidal inclusion given in Equation 25 can be rephrased in a transversely isotropic fourth-rank tensor form

$$
S_{i j k l}=\tilde{F}_{i j k l}\left(S_{1}, S_{2}, S_{3}, S_{4}, S_{5}, S_{6}\right)
$$

where a transversely isotropic fourth-rank tensor $\tilde{\mathbf{F}}$ is defined by six parameters $b_{m}$ ( $m=1$ to 6$)$

$$
\begin{aligned}
\tilde{F}_{i j k l}\left(b_{m}\right)= & b_{1} \tilde{n}_{i} \tilde{n}_{j} \tilde{n}_{k} \tilde{n}_{l}+b_{2}\left(\delta_{i k} \tilde{n}_{j} \tilde{n}_{l}+\delta_{i l} \tilde{n}_{j} \tilde{n}_{k}+\delta_{j k} \tilde{n}_{i} \tilde{n}_{l}+\delta_{j l} \tilde{n}_{i} \tilde{n}_{k}\right) \\
& +b_{3} \delta_{i j} \tilde{n}_{k} \tilde{n}_{l}+b_{4} \delta_{k l} \tilde{n}_{i} \tilde{n}_{j}+b_{5} \delta_{i j} \delta_{k l}+b_{6}\left(\delta_{i k} \delta_{j l}+\delta_{i l} \delta_{j k}\right)
\end{aligned}
$$

with the unit direction vector $\tilde{\mathbf{n}}$ and index $m=1$ to 6 . For a spheroid of $a_{1} \neq a_{2}=a_{3}$, the 1-direction is chosen as symmetric; and therefore, we have $\tilde{n}_{1}=1, \tilde{n}_{2}=\tilde{n}_{3}=0$. In addition, the six parameters on the right-hand side of Equation 34 take the form

$$
\begin{aligned}
& S_{1}=\frac{1}{16} \frac{16+45 \eta+54 \alpha^{2}+60 \eta \alpha^{2}}{\left(\nu_{0}-1\right)\left(1-\alpha^{2}\right)} \\
& S_{2}=\frac{1}{16} \frac{8+15 \eta-8 \nu_{0}-12 \eta \nu_{0}+2 \alpha^{2}+8 \nu_{0} \alpha^{2}+12 \eta \nu_{0} \alpha^{2}}{1-\nu_{0}-\alpha^{2}+\nu_{0} \alpha^{2}} \\
& S_{3}=\frac{1}{16} \frac{3 \eta+10 \alpha^{2}+12 \eta \alpha^{2}}{\left(\nu_{0}-1\right)\left(\alpha^{2}-1\right)} \\
& S_{4}=\frac{1}{16} \frac{3 \eta+16 \nu_{0}+24 \eta \nu_{0}+10 \alpha^{2}+12 \eta \alpha^{2}-16 \nu_{0} \alpha^{2}-24 \nu_{0} \eta \alpha^{2}}{\left(\nu_{0}-1\right)\left(\alpha^{2}-1\right)} \\
& S_{5}=\frac{1}{16} \frac{\eta-8 \eta \nu_{0}-2 \alpha^{2}-4 \eta \alpha^{2}+8 \nu_{0} \eta \alpha^{2}}{\left(\nu_{1}-1\right)\left(\alpha^{2}-1\right)} \\
& S_{6}=\frac{1}{16} \frac{-7 \eta+8 \nu_{0} \eta-2 \alpha^{2}+4 \eta \alpha^{2}-8 \nu_{0} \eta \alpha^{2}}{1-\nu_{0}-\alpha^{2}+\nu_{0} \alpha^{2}}
\end{aligned}
$$

\subsection{EFFECTIVE ELASTOPLASTIC BEHAVIOR OF COMPOSITES WITH ALIGNED DIS- CONTINUOUS FIBERS}

Let us start by considering an initially perfectly bonded two-phase composite consisting of a matrix (phase 0) with bulk modulus $\kappa_{0}$ and shear modulus $\mu_{0}$, and randomly dispersed, aligned spheroidal fibers (phase 1) with bulk modulus $\kappa_{1}$ and shear modulus $\mu_{1}$. When spheroidal inclusions (discontinuous fibers) are aligned in the 1-direction, the composite as a whole is transversely isotropic. Subsequently, as loadings or deformations are applied, some fibers are partially debonded (phase 2), and these partially debonded fibers are regarded as equivalent, transversely isotropic inclusions.

Following Zhao and Weng $(1996,1997)$, a partially debonded isotropic fiber can be replaced by an equivalent, perfectly bonded fiber which possesses yet unknown transversely isotropic moduli. The transverse isotropy of the equivalent fiber can be determined in such a way that: (a) its tensile and shear stresses will 
always vanish in the debonded direction, and (b) its stresses in the bonded directions exist, since the fiber is still able to transmit stresses to the matrix on the bonded surfaces.

When the 1-direction is chosen as symmetric and the plane 2-3 isotropic, the stress-strain relation of a typical transversely isotropic solid can be written as

$$
\left(\begin{array}{c}
\sigma_{11} \\
\sigma_{22} \\
\sigma_{33} \\
\sigma_{23} \\
\sigma_{13} \\
\sigma_{12}
\end{array}\right)=\left[\begin{array}{cccccc}
C_{11} & C_{12} & C_{12} & 0 & 0 & 0 \\
C_{12} & C_{22} & C_{23} & 0 & 0 & 0 \\
C_{12} & C_{23} & C_{22} & 0 & 0 & 0 \\
0 & 0 & 0 & C_{44} & 0 & 0 \\
0 & 0 & 0 & 0 & C_{55} & 0 \\
0 & 0 & 0 & 0 & 0 & C_{55}
\end{array}\right]\left(\begin{array}{c}
\epsilon_{11} \\
\epsilon_{22} \\
\epsilon_{33} \\
2 \epsilon_{23} \\
2 \epsilon_{13} \\
2 \epsilon_{12}
\end{array}\right)
$$

The components of the stiffness matrix take the form

$$
\begin{aligned}
& \frac{C_{22}+C_{23}}{2}=k, C_{12}=l, C_{11}=\bar{n} \\
& \frac{C_{22}-C_{23}}{2}=C_{44}=m, C_{55}=p
\end{aligned}
$$

where $k$ is the plane stress bulk modulus for the lateral dilatation without longitudinal extension $\left(k=\kappa+\frac{\mu}{3}\right)$, $m$ is the rigidity modulus for shearing in any transverse direction, $\bar{n}$ denotes the modulus for the longitudinal uniaxial straining, $l$ denotes the associated cross-modulus, and $p$ signifies the axial shear modulus (Hill, 1964). Therefore, the stress-strain relations for partially debonded composite can be rephrased as

$$
\begin{aligned}
& \frac{1}{2}\left(\sigma_{22}+\sigma_{33}\right)=k\left(\epsilon_{22}+\epsilon_{33}\right)+l \epsilon_{11}, \\
& \sigma_{11}=l\left(\epsilon_{22}+\epsilon_{33}\right)+n \epsilon_{11}, \\
& \sigma_{22}-\sigma_{33}=2 m\left(\epsilon_{22}-\epsilon_{33}\right), \\
& \sigma_{23}=2 m \epsilon_{23}, \sigma_{12}=2 p \epsilon_{12}, \sigma_{13}=2 p \epsilon_{13}
\end{aligned}
$$

It can be easily seen that, by using the inverse of generalized Hook's law, the compliance matrix for a transversely isotropic material may be expressed in the form

$$
\left(\begin{array}{c}
\epsilon_{11} \\
\epsilon_{22} \\
\epsilon_{33} \\
2 \epsilon_{23} \\
2 \epsilon_{13} \\
2 \epsilon_{12}
\end{array}\right)=\left[\begin{array}{cccccc}
\frac{k}{l^{2}+k n} & \frac{l}{2\left(l^{2}-k n\right)} & \frac{l}{2\left(l^{2}-k n\right)} & 0 & 0 & 0 \\
\frac{l}{2\left(l^{2}-k n\right)} & \frac{-l^{2}+k n+m n}{4 m\left(-l^{2}+k n\right)} & \frac{l^{2}-k n+m n}{4 m\left(-l^{2}+k n\right)} & 0 & 0 & 0 \\
\frac{l}{2\left(l^{2}-k n\right)} & \frac{l^{2}-k n+m n}{4 m\left(-l^{2}+k n\right)} & \frac{-l^{2}+k n+m n}{4 m\left(-l^{2}+k n\right)} & 0 & 0 & 0 \\
0 & 0 & 0 & \frac{1}{m} & 0 & 0 \\
0 & 0 & 0 & 0 & \frac{1}{p} & 0 \\
0 & 0 & 0 & 0 & 0 & \frac{1}{p}
\end{array}\right]\left(\begin{array}{c}
\sigma_{11} \\
\sigma_{22} \\
\sigma_{33} \\
\sigma_{23} \\
\sigma_{13} \\
\sigma_{12}
\end{array}\right)
$$


For the special case of uniaxial loading, Equation 45 can be simplified as

$$
\epsilon_{i j}=\left[\begin{array}{ccc}
\frac{k}{-l^{2}+k n} & 0 & 0 \\
0 & \frac{l}{2\left(l^{2}-k n\right)} & 0 \\
0 & 0 & \frac{l}{2\left(l^{2}-k n\right)}
\end{array}\right] \sigma_{11}
$$

The transversely isotropic fiber can be considered to be under the condition of plane stress with the components in the 1-direction being zero. To ensure the equivalence between a partially debonded isotropic fiber and an equivalent, perfectly bonded transversely isotropic fiber, the elastic moduli of a transversely isotropic fiber, with the condition $\sigma_{11}=\sigma_{12}=\sigma_{13}=0$, can be derived as

$$
k_{2}=\frac{\mu_{1}\left(3 k_{1}-\mu_{1}\right)}{k_{1}+\mu_{1}}, \quad l_{2}=0, \quad n_{2}=0, \quad m_{2}=\mu_{1}, \quad p_{2}=0
$$

where the subscripts 1 and 2 refer to phases 1 and 2 moduli, respectively.

In accordance with the notation given in Equation 43 and the the transversely isotropic fourth-rank tensor $\tilde{\mathbf{F}}$ defined in Equation 35, the stiffness tensor $\mathbf{C}_{2}$ for the equivalent, transversely isotropic fiber can be represented as

$$
\mathbf{C}_{2}=\tilde{F}_{i j k l}\left(t_{1}, t_{2}, t_{3}, t_{4}, t_{5}, t_{6}\right)
$$

where the six parameters on the right-hand side take the form

$$
\begin{aligned}
& t_{1}=k_{2}+n_{2}+m_{2}-4 p_{2}-2 l_{2} \\
& t_{2}=-m_{2}+p_{2} \\
& t_{3}=-k_{2}+m_{2}+l_{2} \\
& t_{4}=-k_{2}+m_{2}+l_{2} \\
& t_{5}=k_{2}-m_{2} \\
& t_{6}=m_{2}
\end{aligned}
$$

The relationship between the stress tensor $\boldsymbol{\sigma}$ and the strain tensor $\boldsymbol{\epsilon}$ at any point $\mathbf{x}$ in the $q$-phase $(q=$ $0,1,2)$ is governed by

$$
\boldsymbol{\sigma}(\mathbf{x})=\mathbf{C}_{q}: \epsilon(\mathbf{x})
$$

where ":" denotes the tensor contraction and $\mathbf{C}_{q}$ is the elasticity tensor of the $q$-phase.

Effective elastic moduli of multi-phase composites containing randomly located, unidirectionally aligned elastic ellipsoids were explicitly derived by Ju and Chen (1994a) accounting for far-field perturbations. For a multi-phase composite, the effective (noninteracting) elasticity tensor $\mathbf{C}_{*}$ reads

$$
\mathbf{C}_{*}=\mathbf{C}_{0} \cdot\left\{\mathbf{I}+\mathbf{B} \cdot(\mathbf{I}-\mathbf{S} \cdot \mathbf{B})^{-1}\right\}
$$


where $\mathbf{I}$ is the fourth-rank identity tensor, "." denotes the tensor multiplication and $\mathbf{B}$ takes the form

$$
\mathbf{B}=\sum_{q=1}^{n} \phi_{q}\left(\mathbf{S}+\mathbf{A}_{q}\right)^{-1}
$$

Here, $n$ signifies the number of inclusion phases of different material properties, and $\phi_{q}$ denotes the volume fraction of the $q$-phase. In addition, the fourth-rank tensor $\mathbf{A}_{q}$ is defined as

$$
\mathbf{A}_{q}=\left[\mathbf{C}_{q}-\mathbf{C}_{0}\right]^{-1} \cdot \mathbf{C}_{0}
$$

Accordingly, in the case of aligned (in the $x_{1}$-direction) fiber-reinforced composites, the effective elastic stiffness tensor $\mathbf{C}_{*}$ can be explicitly derived as

$$
\mathbf{C}_{*}=C_{I K}^{(1)} \delta_{i j} \delta_{k l}+C_{I J}^{(2)}\left(\delta_{i k} \delta_{j l}+\delta_{i l} d_{j k}\right)
$$

where

$$
\begin{aligned}
C_{I K}^{(1)} & =\lambda_{0}-\sum_{r=1}^{2}\left(2 \mu_{0} \phi_{r} \frac{\left(\Psi_{r}\right)_{I K}}{\left(\psi_{r}\right)_{I I}}+\frac{\lambda_{0} \phi_{r}}{\left(\psi_{r}\right)_{K K}}-\lambda_{0} \phi_{r} \sum_{m=1}^{3} \frac{\left(\Psi_{r}\right)_{m K}}{\left(\psi_{r}\right)_{m m}}\right) \\
C_{I J}^{(2)} & =\mu_{0} \sum_{r=1}^{2}\left(1+\frac{\phi_{r}}{\left(\psi_{r}\right)_{I J}}\right)
\end{aligned}
$$

with

$$
\begin{aligned}
& \left(\psi_{r}\right)_{I J}=2\left[\left(Z_{r}\right)_{2}+\left(1-\phi_{r}\right) S_{I J}^{(2)}\right] \\
& \left(\Psi_{r}\right)_{I 1}=\frac{\left(\xi_{r}\right)_{2}\left[\left(Z_{r}\right)_{1}+\left(1-\phi_{r}\right) S_{I 1}^{(1)}\right]-2\left(\xi_{r}\right)_{4}\left[\left(Z_{r}\right)_{1}+\left(1-\phi_{r}\right) S_{I 2}^{(1)}\right]}{\left(\xi_{r}\right)_{1}\left(\xi_{r}\right)_{2}-2\left(\xi_{r}\right)_{3}\left(\xi_{r}\right)_{4}} \\
& \left(\Psi_{r}\right)_{I 2}=\left(\Psi_{r}\right)_{I 3}=\frac{\left(\xi_{r}\right)_{1}\left[\left(Z_{r}\right)_{1}+\left(1-\phi_{r}\right) S_{I 2}^{(1)}\right]-\left(\xi_{r}\right)_{3}\left[\left(Z_{r}\right)_{1}+\left(1-\phi_{r}\right) S_{I 1}^{(1)}\right]}{\left(\xi_{r}\right)_{1}\left(\xi_{r}\right)_{2}-2\left(\xi_{r}\right)_{3}\left(\xi_{r}\right)_{4}}
\end{aligned}
$$

and

$$
\begin{aligned}
& \left(\xi_{r}\right)_{1}=\left(Z_{r}\right)_{1}+\left(1-\phi_{r}\right) S_{11}^{(1)}+\left(\psi_{r}\right)_{11} \\
& \left(\xi_{r}\right)_{2}=2\left(Z_{r}\right)_{1}+2\left(1-\phi_{r}\right) S_{22}^{(1)}+\left(\psi_{r}\right)_{22} \\
& \left(\xi_{r}\right)_{3}=\left(Z_{r}\right)_{1}+\left(1-\phi_{r}\right) S_{12}^{(1)} \\
& \left(\xi_{r}\right)_{4}=\left(Z_{r}\right)_{1}+\left(1-\phi_{r}\right) S_{21}^{(1)}
\end{aligned}
$$

where the components of Eshelby's tensor $S_{11}^{(1)}, \ldots, S_{21}^{(1)}$ are given in Equations 26-32 and

$$
\begin{aligned}
\left(Z_{r}\right)_{1} & =\frac{\lambda_{0} \mu_{r}-\lambda_{r} \mu_{0}}{\left(\mu_{r}-\mu_{0}\right)\left[2\left(\mu_{r}-\mu_{0}\right)+3\left(\lambda_{r}-\lambda_{0}\right)\right]} \\
\left(Z_{r}\right)_{2} & =\frac{\mu_{0}}{2\left(\mu_{r}-\mu_{0}\right)}
\end{aligned}
$$


It is noted that $C_{12}^{(1)}=C_{13}^{(1)}, C_{21}^{(1)}=C_{31}^{(1)}, C_{22}^{(1)}=C_{23}^{(1)}=C_{32}^{(1)}=C_{33}^{(1)}, C_{12}^{(2)}=C_{21}^{(2)}=C_{13}^{(2)}=C_{31}^{(2)}, C_{22}^{(2)}=$ $C_{33}^{(2)}=C_{23}^{(2)}=C_{32}^{(2)}$. Thus, as expected, the overall elastic stiffness tensor $\mathbf{C}_{*}$ in Equation 59 for aligned fiber-reinforced composites is transversely isotropic.

We now consider the overall elastoplastic responses of progressively debonded fiber-reinforced composites, which initially feature perfect interfacial bonding between fibers and the matrix in two-phase composites. It is known that partial interfacial debonding may occur in some fibers under applied loading. Therefore, an original two-phase composite may gradually become a three-phase composite consisting of the matrix, perfectly bonded fibers, and partially debonded fibers. In what follows, for simplicity, we will regard partially debonded fibers as equivalent, perfectly bonded transversely isotropic fibers. Also for simplicity, the von Mises yield criterion with isotropic hardening law is assumed. Extension of the present framework to general yield criterion and the general hardening law, nevertheless, is straightforward. Accordingly, at any matrix material point, the stress $\boldsymbol{\sigma}$ and the equivalent plastic strain $\bar{e}^{p}$ must satisfy the following yield function:

$$
F\left(\boldsymbol{\sigma}, \bar{e}^{p}\right)=H(\boldsymbol{\sigma})-K^{2}\left(\bar{e}^{p}\right) \leq 0
$$

in which $K\left(\bar{e}^{p}\right)$ is the isotropic hardening function of the matrix-only material. Furthermore, $H(\boldsymbol{\sigma}) \equiv \boldsymbol{\sigma}: \mathbf{I}_{d}$ : $\boldsymbol{\sigma}$ denotes the square of the deviatoric stress norm, where $\mathbf{I}_{d}$ signifies the deviatoric part of the fourth-rank identity tensor $\mathbf{I}$

$$
\mathbf{I}_{d} \equiv \mathbf{I}-\frac{1}{3} \mathbf{1} \otimes \mathbf{1}
$$

in which 1 represents the second-rank identity tensor and " $\otimes$ " denotes the tensor expansion.

According to the theory of continuum plasticity, the total strain $\epsilon$ can be decomposed into two parts

$$
\boldsymbol{\epsilon}=\boldsymbol{\epsilon}^{e}+\boldsymbol{\epsilon}^{p}
$$

where $\boldsymbol{\epsilon}^{e}$ is the elastic strain of the matrix or fibers, and $\boldsymbol{\epsilon}^{p}$ represents the stress-free plastic strain in the plastic matrix only. In addition, elastic response on elastic stain is defined as

$$
\overline{\boldsymbol{\sigma}}=\mathbf{C}_{*}: \overline{\boldsymbol{\epsilon}}^{e}
$$

In order to solve the elastoplastic response exactly, the stress at any local point has to be solved and then used to determine the plastic response through the local yield criterion for all possible configurations. This approach is generally infeasible due to the complexity of statistical and microstructural information. Therefore, we propose a framework in which an ensemble-averaged yield criterion is constructed for the entire composite. The methodology is generally parallel to the work of Ju and Chen (1994a) and Ju and Lee (1999) in which only the first order effects are considered in the formulation of effective plastic response. 
In the following, small strains are assumed; and therefore, the statistical microstructure of fibers embedded in a ductile matrix remains essentially the same. Hence, the microstructure is taken as statistically homogeneous and isotropic with a virtually constant volume fraction for the summation of perfectly bonded fibers and partially debonded fibers during the deformation process. Furthermore, both perfectly bonded fibers and partially debonded fibers are considered as spheroids of uniform size. The extension to accommodate different or distributed sizes of fibers is readily feasible.

Following Ju and Lee (1999), we denote, by $H(\mathbf{x} \mid \mathcal{G})$, the square of the "current stress norm" at the local point $\mathbf{x}$, which determines the plastic strain in a composite for a given phase configuration $\mathcal{G}$. Since there is no plastic strain in the elastic perfectly bonded fibers or partially debonded fibers, $H(\mathbf{x} \mid \mathcal{G})$ can be written as

$$
H(\mathbf{x} \mid \mathcal{G})=\left\{\begin{array}{cl}
\boldsymbol{\sigma}(\mathbf{x} \mid \mathcal{G}): \mathbf{I}_{d}: \boldsymbol{\sigma}(\mathbf{x} \mid \mathcal{G}), & \text { if } \mathbf{x} \text { in the matrix } \\
0, & \text { otherwise }
\end{array}\right.
$$

In addition, $\langle H\rangle_{m}(\mathbf{x})$ is defined as the ensemble average of $H(\mathbf{x} \mid \mathcal{G})$ over all possible realizations where $\mathbf{x}$ is in the matrix phase. Here, the angled bracket $\langle\cdot\rangle$ signifies the ensemble average operator. Let $P\left(\mathcal{G}_{q}\right)$ be the probability density function for finding the $q$-phase $(q=1,2)$ configuration $\mathcal{G}_{q}$ in the composite. $\langle H\rangle_{m}(\mathbf{x})$ can be obtained by integrating $H$ over all possible perfectly bonded fibers and partially debonded fibers configurations (for a point $\mathbf{x}$ in the matrix)

$$
\langle H\rangle_{m}(\mathbf{x})=H^{o}+\int_{\mathcal{G}_{1}}\left\{H\left(\mathbf{x} \mid \mathcal{G}_{1}\right)-H^{o}\right\} P\left(\mathcal{G}_{1}\right) d \mathcal{G}+\int_{\mathcal{G}_{2}}\left\{H\left(\mathbf{x} \mid \mathcal{G}_{2}\right)-H^{o}\right\} P\left(\mathcal{G}_{2}\right) d \mathcal{G}
$$

where $H^{o}$ is the square of the far-field stress norm in the matrix:

$$
H^{o}=\boldsymbol{\sigma}^{o}: \mathbf{I}_{d}: \boldsymbol{\sigma}^{o}
$$

Moreover, the total stress at any point $\mathbf{x}$ in the matrix is the superposition of the far-field stress $\boldsymbol{\sigma}^{o}$ and the perturbed stress $\boldsymbol{\sigma}^{\prime}$ due to the presence of the particles and voids:

$$
\sigma(\mathbf{x})=\sigma^{o}+\sigma^{\prime}(\mathbf{x})
$$

in which $\boldsymbol{\sigma}^{o}$ and $\boldsymbol{\sigma}^{\prime}$ are defined as

$$
\begin{gathered}
\boldsymbol{\sigma}^{o} \equiv \mathbf{C}_{0}: \boldsymbol{\epsilon}^{o} \\
\boldsymbol{\sigma}^{\prime}(\mathbf{x}) \equiv \mathbf{C}_{0}: \int_{V} \mathbf{G}\left(\mathbf{x}-\mathbf{x}^{\prime}\right): \boldsymbol{\epsilon}_{1}^{*}\left(\mathbf{x}^{\prime}\right) d \mathbf{x}^{\prime}+\mathbf{C}_{0}: \int_{V} \mathbf{G}\left(\mathbf{x}-\mathbf{x}^{\prime}\right): \epsilon_{2}^{*}\left(\mathbf{x}^{\prime}\right) d \mathbf{x}^{\prime}
\end{gathered}
$$

where $\boldsymbol{\epsilon}^{o}$ is the elastic strain field induced by the far-field loading, $\boldsymbol{\epsilon}_{q}^{*}\left(\mathbf{x}^{\prime}\right)$ denotes the elastic eigenstrain in the $q$-phase ( $q=1,2$ only), and $\mathbf{x}^{\prime}$ resides in either a perfectly bonded fiber or a partially debonded fiber. 
Further, $\mathbf{C}_{0}$ denotes the fourth-rank elasticity tensor of the matrix, and $V$ is the statistically representative volume element.

The unknown elastic eigenstrain $\boldsymbol{\epsilon}_{q}^{*}(\mathbf{x})$ within the $q$-phase can be solved by the integral equation obtained from Eshelby's equivalence principle (Eshelby, 1957). The outcome is

$$
-\mathbf{A}_{q}: \boldsymbol{\epsilon}_{q}^{*}(\mathbf{x})=\boldsymbol{\epsilon}^{o}+\int_{V} \mathbf{G}\left(\mathbf{x}-\mathbf{x}^{\prime}\right): \boldsymbol{\epsilon}_{q}^{*}\left(\mathbf{x}^{\prime}\right) d \mathbf{x}^{\prime}
$$

where the fourth-rank tensor $\mathbf{A}_{q}$ is defined in Equation 58.

According to Eshelby (1957), the (elastic) eigenstrain for a single ellipsoidal inclusion is uniform for the interior points of an isolated (i.e., noninteracting) inclusion. Consequently, the constant (elastic) eigenstrain can be moved out of the integral in Equation 80 in accordance with the first-order approximation approach proposed by Ju and Chen (1994a). It is noted that, for the first-order approximation method, the interactions among fibers are neglected in the process of collecting the perturbations of stresses at a local matrix point for the purpose of predicting the plastic behavior. Therefore, the perturbed stress for any matrix point $\mathbf{x}$ due to a typical isolated $q$-phase inhomogeneity centered at $\mathbf{x}_{q}^{(1)}$ takes the form

$$
\boldsymbol{\sigma}^{\prime}\left(\mathbf{x} \mid \mathbf{x}_{q}^{(1)}\right)=\left[\mathbf{C}_{0} \cdot \overline{\mathbf{G}}\left(\mathbf{x}-\mathbf{x}_{q}^{(1)}\right)\right]: \boldsymbol{\epsilon}_{q}^{* o}
$$

where $\boldsymbol{\epsilon}_{q}^{* o}$ is the solution of the (elastic) eigenstrain $\boldsymbol{\epsilon}_{q}^{*}$ for the single inclusion problem of the $q$-phase, and

$$
\overline{\mathbf{G}}\left(\mathbf{x}-\mathbf{x}_{q}^{(1)}\right) \equiv \int_{\Omega_{q}^{(1)}} \mathbf{G}\left(\mathbf{x}-\mathbf{x}^{\prime}\right) d \mathbf{x}^{\prime}
$$

for $\mathbf{x} \notin \Omega_{q}^{(1)}$ in which $\Omega_{q}^{(1)}$ is the single inhomogeneity domain centered at $\mathbf{x}_{q}^{(1)}$ in the $q$-phase.

Moreover, the elastic "noninteracting" eigenstrain $\boldsymbol{\epsilon}_{q}^{* o}$ in Equation 82 is given by

$$
\boldsymbol{\epsilon}_{q}^{* o}=-\left(\mathbf{A}_{q}+\mathbf{S}\right)^{-1}: \boldsymbol{\epsilon}^{o}, \quad q=1,2
$$

where $\mathbf{S}$ is Eshelby's tensor for a spheroidal inclusion (see Ju and Chen 1994a and 1994b for example).

As indicated before, a matrix point receives the perturbations from perfectly bonded fibers and partially debonded fibers. Therefore, the ensemble-average stress norm for any matrix point $\mathbf{x}$ can be evaluated by collecting and summing up all the current stress norm perturbations produced by any typical perfectly bonded fiber centered at $\mathbf{x}_{1}^{(1)}$ in the perfectly bonded fiber domain and any typical partially debonded fiber centered at $\mathbf{x}_{2}^{(1)}$ in the partially debonded fiber domain, and averaging over all possible locations of $\mathbf{x}_{1}^{(1)}$ and $\mathbf{x}_{2}^{(1)}$. As a result, we arrive at

$$
\langle H\rangle_{m}(\mathbf{x}) \cong H^{o}+\int_{\mathbf{x}_{1}^{(1)} \notin \Xi(\mathbf{x})}\left\{H\left(\mathbf{x} \mid \mathbf{x}_{1}^{(1)}\right)-H^{o}\right\} P\left(\mathbf{x}_{1}^{(1)}\right) d \mathbf{x}_{1}^{(1)}
$$




$$
+\int_{\mathbf{x}_{2}^{(1)} \notin \Xi(\mathbf{x})}\left\{H\left(\mathbf{x} \mid \mathbf{x}_{2}^{(1)}\right)-H^{o}\right\} P\left(\mathbf{x}_{2}^{(1)}\right) d \mathbf{x}_{2}^{(1)}+\ldots .
$$

where $\Xi_{\mathbf{x}}$ is the exculsion zone and $P\left(\mathbf{x}_{1}^{(1)}\right)$ and $P\left(\mathbf{x}_{2}^{(1)}\right)$ denote the probability density functions for finding a perfectly bonded fiber centered at $\mathbf{x}_{1}^{(1)}$ and a partially debonded fiber centered at $\mathbf{x}_{2}^{(1)}$, respectively. Here, for simplicity, $P\left(\mathbf{x}_{1}^{(1)}\right)$ and $P\left(\mathbf{x}_{2}^{(1)}\right)$ are assumed to be statistically homogeneous, isotropic and uniform. That is, we assume that the probability density functions take the form $P\left(\mathbf{x}_{1}^{(1)}\right)=\frac{N_{1}}{V}$ and $P\left(\mathbf{x}_{2}^{(1)}\right)=\frac{N_{2}}{V}$, where $N_{1}$ and $N_{2}$ are the total numbers of perfectly bonded fibers and partially debonded fibers, respectively, dispersed in a representative volume $V$. We define a tiny equal-volume spherical probabilistic zone with the radius $a^{*}=\left(a_{1} a_{2}^{2}\right)^{1 / 3}$, or $a^{*}=a_{1} / \alpha^{2 / 3}$, where $\alpha=a_{1} / a_{2}$ is the aspect ratio (the ratio of length to diameter) of a spheroid. Further, owing to the assumption of statistical isotropy and uniformity Equation 85 can be recast into a more convenient form:

$$
\begin{aligned}
\langle H\rangle_{m}(\mathbf{x}) \cong & H^{o}+\frac{N_{1}}{V} \int_{\hat{r}_{1}>a^{*}} d \hat{r}_{1} \int_{A\left(\hat{r}_{1}\right)}\left\{H\left(\hat{\mathbf{r}}_{1}\right)-H^{o}\right\} d A \\
& +\frac{N_{2}}{V} \int_{\hat{r}_{2}>a^{*}} d \hat{r}_{2} \int_{A\left(\hat{r}_{2}\right)}\left\{H\left(\hat{\mathbf{r}}_{2}\right)-H^{o}\right\} d A+\ldots
\end{aligned}
$$

where $A\left(\hat{r}_{q}\right)$ is a spherical surface of radius $\hat{r}_{q}(q=1,2)$ in the probability space.

Upon integrating the surface integration for ensemble average, the following two identities are needed

$$
\begin{aligned}
& \int_{A\left(\hat{r}_{q}\right)} \hat{n}_{i} \hat{n}_{j} d A=\frac{4 \pi \hat{r}_{q}^{2}}{3} \Delta_{I} \delta_{i j} \\
& \int_{A\left(\hat{r}_{q}\right)} \hat{n}_{i} \hat{n}_{j} \hat{n}_{k} \hat{n}_{l} d A=\frac{4 \pi \hat{r}_{q}^{2}}{15}\left[\Delta_{I K} \delta_{i j} \delta_{k l}+\Delta_{I J}\left(\delta_{i k} \delta_{j l}+\delta_{i l} \delta_{j k}\right)\right]
\end{aligned}
$$

where the components of $\Delta_{I}$ and $\Delta_{I J}$ read:

$$
\begin{aligned}
& \Delta_{1}=\frac{3\left[1-\alpha^{4} f\left(\alpha^{2}\right)\right]}{1-\alpha^{4}} \\
& \Delta_{2}=\Delta_{3}=\frac{1}{2}\left(3-\Delta_{1}\right) \\
& \Delta_{I J}=\left[\begin{array}{lll}
b & c & c \\
c & d & d \\
c & d & d
\end{array}\right]
\end{aligned}
$$

with

$$
f(\alpha)= \begin{cases}\frac{\cos ^{-1} \alpha}{\alpha \sqrt{1-\alpha^{2}}}, & \alpha<1 \\ \frac{\cosh ^{-1} \alpha}{\alpha \sqrt{\alpha^{2}-1}}, & \alpha>1\end{cases}
$$

and

$$
b=\frac{5}{2\left(1-\alpha^{4}\right)^{2}}\left[2+\alpha^{4}-3 \alpha^{4} f\left(\alpha^{2}\right)\right]
$$




$$
\begin{aligned}
c & =\frac{15 \alpha^{4}}{4\left(1-\alpha^{4}\right)^{2}}\left[-3+\left(1+2 \alpha^{4}\right) f\left(\alpha^{2}\right)\right] \\
d & =\frac{1}{8}(15-3 b-4 c)
\end{aligned}
$$

With the help of two identities in Equations 87-88 and by dropping higher order terms, the integral on the right-hand side of Equation 86 can be evaluated and we arrive at the ensemble-averaged current stress norm at any matrix point

$$
\langle H\rangle_{m}(\mathbf{x})=\boldsymbol{\sigma}^{o}: \mathbf{T}: \boldsymbol{\sigma}^{o}
$$

The components of the positive definite fourth-rank tensor $\mathbf{T}$ read

$$
T_{i j k l}=T_{I K}^{(1)} \delta_{i j} \delta_{k l}+T_{I J}^{(2)}\left(\delta_{i k} \delta_{j l}+\delta_{i l} \delta_{j k}\right)
$$

where

$$
\begin{aligned}
T_{I K}^{(1)}= & -\frac{1}{3}+\sum_{r=1}^{2} \frac{2 \phi_{r}}{4725\left(1-\nu_{0}\right)^{2}\left(B_{r}\right)_{I I}\left(B_{r}\right)_{K K}}\left\{1575\left(1-2 \nu_{0}\right)^{2}\left(\Lambda_{r}\right)_{I I}\left(\Lambda_{r}\right)_{K K}\right. \\
& +21\left(25 \nu_{0}-23\right)\left(1-2 \nu_{0}\right)\left[\left(\Lambda_{r}\right)_{I I} \Delta_{K}+\left(\Lambda_{r}\right)_{K K} \Delta_{I}\right] \\
& +21\left(25 \nu_{0}-2\right)\left(1-2 \nu_{0}\right)\left[\left(\Lambda_{r}\right)_{I I}+\left(\Lambda_{r}\right)_{K K}\right]+3\left(35 \nu_{0}^{2}-70 \nu_{0}+36\right) \Delta_{I K} \\
& \left.+7\left(50 \nu_{0}^{2}-59 \nu_{0}+8\right)\left(\Delta_{I}+\Delta_{K}\right)-2\left(175 \nu_{0}^{2}-343 \nu_{0}+103\right)\right\} \\
T_{I J}^{(2)}= & \frac{1}{2}+\sum_{r=1}^{2} \frac{\phi_{r}}{1575\left(1-\nu_{0}\right)^{2}\left(B_{r}\right)_{I J}\left(B_{r}\right)_{I J}}\left\{\left(72-140 \nu_{0}+70 \nu_{0}^{2}\right) \Delta_{I J}\right. \\
& \left.-\left(75-266 \nu_{0}+175 \nu_{0}^{2}\right) \frac{\Delta_{I}+\Delta_{J}}{2}+164-476 \nu_{0}+350 \nu_{0}^{2}\right\}
\end{aligned}
$$

in which the (current) volume fraction for $r$-phase is defined as $\phi_{r}=\frac{4 \pi\left(a^{*}\right)^{3}}{3} \frac{N_{r}}{V}$. Further, we have

$$
\begin{aligned}
& \left(B_{r}\right)_{I J}=2\left[\left(Z_{r}\right)_{2}+\mathcal{S}_{I J}^{(2)}\right] \\
& \left(\Lambda_{r}\right)_{I 1}=\frac{\left(\mathcal{X}_{r}\right)_{I 1}}{\left(\tilde{\mathcal{X}}_{r}\right)} \\
& \left(\Lambda_{r}\right)_{I 2}=\left(\Lambda_{r}\right)_{I 3}=\frac{\left(\mathcal{X}_{r}\right)_{I 2}}{\left(\tilde{\mathcal{X}}_{r}\right)}
\end{aligned}
$$

where

$$
\begin{aligned}
& \left(\mathcal{X}_{r}\right)_{I 1}=\left[2\left(Z_{r}\right)_{1}+2 \mathcal{S}_{22}^{(1)}+\left(B_{r}\right)_{22}\right]\left[\left(Z_{r}\right)_{1}+\mathcal{S}_{I 1}^{(1)}\right]-2\left[\left(Z_{r}\right)_{1}+\mathcal{S}_{21}^{(1)}\right]\left[\left(Z_{r}\right)_{1}+\mathcal{S}_{I 2}^{(1)}\right] \\
& \left(\tilde{\mathcal{X}}_{r}\right)=\left[2\left(Z_{r}\right)_{1}+2 \mathcal{S}_{22}^{(1)}+\left(B_{r}\right)_{22}\right]\left[\left(Z_{r}\right)_{1}+\mathcal{S}_{11}^{(1)}+\left(B_{r}\right)_{11}\right]-2\left[\left(Z_{r}\right)_{1}+\mathcal{S}_{12}^{(1)}\right]\left[\left(Z_{r}\right)_{1}+\mathcal{S}_{21}^{(1)}\right] \\
& \left(\mathcal{X}_{r}\right)_{I 2}=\left[\left(Z_{r}\right)_{1}+\mathcal{S}_{11}^{(1)}+\left(B_{r}\right)_{11}\right]\left[\left(Z_{r}\right)_{1}+\mathcal{S}_{I 2}^{(1)}\right]-\left[\left(Z_{r}\right)_{1}+\mathcal{S}_{12}^{(1)}\right]\left[\left(Z_{r}\right)_{1}+\mathcal{S}_{I 1}^{(1)}\right]
\end{aligned}
$$

The ensemble-averaged current stress norm at a matrix point can also be expressed in terms of the macroscopic stress $\overline{\boldsymbol{\sigma}}$. Following Ju and Chen (1994a), the relation between the far-field stress $\boldsymbol{\sigma}^{o}$ and the macroscopic stress $\overline{\boldsymbol{\sigma}}$ takes the form

$$
\boldsymbol{\sigma}^{o}=\mathbf{P}: \overline{\boldsymbol{\sigma}}
$$


where the fourth-rank tensor $\mathbf{P}$ reads

$$
\mathbf{P}=\mathbf{I}+\phi_{1}(\mathbf{I}-\mathbf{S}) \cdot\left(\mathbf{A}_{1}+\mathbf{S}\right)^{-1}+\phi_{2}(\mathbf{I}-\mathbf{S}) \cdot\left(\mathbf{A}_{2}+\mathbf{S}\right)^{-1}
$$

and the components of $\mathbf{P}$ are

$$
P_{i j k l}=P_{I K}^{(1)} \delta_{i j} \delta_{k l}+P_{I J}^{(2)}\left(\delta_{i k} \delta_{j l}+\delta_{i l} \delta_{j k}\right)
$$

with

$$
\begin{aligned}
& P_{I K}^{(1)}=-\frac{\Gamma_{I K}^{(3)}}{2 \Gamma_{I I}^{(2)}} \\
& P_{I J}^{(2)}=\frac{1}{4 \Gamma_{I J}^{(2)}}
\end{aligned}
$$

and the coefficients $\Gamma_{I K}^{(3)}, \Gamma_{I I}^{(2)}$ and $\Gamma_{I J}^{(2)}$ read:

$$
\begin{aligned}
\Gamma_{I 1}^{(3)} & =\frac{\left.\Gamma_{22}^{(1)}+\Gamma_{22}^{(2)}\right) \Gamma_{I 1}^{(1)}-\Gamma_{21}^{(1)} \Gamma_{I 2}^{(1)}}{\left(\Gamma_{11}^{(1)}+2 \Gamma_{11}^{(2)}\right)\left(\Gamma_{22}^{(1)}+\Gamma_{22}^{(2)}\right)-\Gamma_{12}^{(1)} \Gamma_{21}^{(1)}} \\
\Gamma_{I 2}^{(3)} & =\Gamma_{I 3}^{(3)}=\frac{\left(\Gamma_{11}^{(1)}+2 \Gamma_{11}^{(2)}\right) \Gamma_{I 2}^{(1)}-\Gamma_{12}^{(1)} \Gamma_{I 1}^{(1)}}{2\left(\Gamma_{11}^{(1)}+2 \Gamma_{11}^{(2)}\right)\left(\Gamma_{22}^{(1)}+\Gamma_{22}^{(2)}\right)-2 \Gamma_{12}^{(1)} \Gamma_{21}^{(1)}}
\end{aligned}
$$

and

$$
\begin{aligned}
& \Gamma_{I J}^{(2)}=\frac{1}{2}+\sum_{r=1}^{2} \frac{\left(1-2 \mathcal{S}_{I J}^{(2)}\right) \phi_{r}}{2\left(B_{r}\right)_{I J}} \\
& \Gamma_{I K}^{(1)}=\sum_{r=1}^{2} \phi_{r}\left[\sum_{m=1}^{3} \frac{\left(\Lambda_{r}\right)_{m K} \mathcal{S}_{I m}^{(1)}}{\left(B_{r}\right)_{m m}}-\frac{\mathcal{S}_{I K}^{(1)}}{\left(B_{r}\right)_{K K}}-\frac{\left(\Lambda_{r}\right)_{I K}\left(1-2 \mathcal{S}_{I I}^{(2)}\right)}{\left(B_{r}\right)_{I I}}\right]
\end{aligned}
$$

By combining Equations 96 and 106, we arrive at the alternative expression for the ensemble-averaged current stress norm (square) in a matrix point:

$$
\langle H\rangle_{m}(\mathbf{x})=\overline{\boldsymbol{\sigma}}: \overline{\mathbf{T}}: \overline{\boldsymbol{\sigma}}
$$

where the positive definite fourth-rank tensor $\overline{\mathbf{T}}$ is defined as

$$
\overline{\mathbf{T}} \equiv \mathbf{P}^{\mathbf{T}} \cdot \mathbf{T} \cdot \mathbf{P}
$$

and can be shown to be

$$
\bar{T}_{i j k l}=\bar{T}_{I K}^{(1)} \delta_{i j} \delta_{k l}+\bar{T}_{I J}^{(2)}\left(\delta_{i k} \delta_{j l}+\delta_{i l} \delta_{j k}\right)
$$

where

$$
\bar{T}_{I K}^{(1)}=4 P_{I I}^{(2)} T_{I I}^{(2)} P_{I K}^{(1)}+4 P_{K K}^{(2)} T_{K K}^{(2)} P_{K I}^{(1)}+4 P_{I I}^{(2)} T_{I K}^{(1)} P_{K K}^{(2)}
$$




$$
\begin{aligned}
& +\sum_{m=1}^{3} 2 P_{I I}^{(2)} T_{I m}^{(1)} P_{m K}^{(1)}+\sum_{m=1}^{3} 2 P_{K K}^{(2)} T_{m K}^{(1)} P_{m I}^{(1)} \\
& +2 \sum_{m=1}^{3} \sum_{n=1}^{3}\left(P_{m K}^{(1)} T_{m m}^{(2)} P_{m I}^{(1)}+P_{m K}^{(1)} T_{n m}^{(1)} P_{n I}^{(1)}\right) \\
\bar{T}_{I J}^{(2)}= & 4 P_{I J}^{(2)} T_{I J}^{(2)} P_{I J}^{(2)}
\end{aligned}
$$

The ensemble-volume averaged "current stress norm" for any point $\mathrm{x}$ in a three-phase fiber-reinforced composite can be defined as:

$$
\sqrt{\langle H\rangle(\mathbf{x})}=\left(1-\phi_{1}\right) \sqrt{\overline{\boldsymbol{\sigma}}: \overline{\mathbf{T}}: \overline{\boldsymbol{\sigma}}}
$$

where $\phi_{1}$ is the current perfectly bonded fiber volume fraction. Therefore, the effective yield function for the three-phase fiber-reinforced composite can be proposed as

$$
\bar{F}=\left(1-\phi_{1}\right)^{2} \overline{\boldsymbol{\sigma}}: \overline{\mathbf{T}}: \overline{\boldsymbol{\sigma}}-K^{2}\left(\bar{e}^{p}\right)
$$

where the isotropic hardening function $K\left(\bar{e}^{p}\right)$ is defined as

$$
K\left(\bar{e}^{p}\right)=\sqrt{\frac{2}{3}}\left\{\sigma_{y}+h\left(\bar{e}^{p}\right)^{\bar{q}}\right\}
$$

where $\sigma_{y}$ is the initial yield stress, and $h$ and $\bar{q}$ signify the linear and exponential isotropic hardening parameters, respectively, for the three-phase composite.

\subsection{EFFECTIVE ELASTOPLASTIC BEHAVIOR OF COMPOSITES WITH RANDOMLY ORIENTED FIBERS}

Consider the composite model depicted in Figure 3, in which spheroidal fibers with an aspect ratio of $\alpha$ (the ratio of length to diameter) are uniformly dispersed and randomly oriented in three-dimensional space. The average process over all orientations upon governing constitutive field equations is performed to obtain the constitutive relations and the overall yield function for the RFPCs. The overall plastic flow rule and hardening law, with the proposed overall yield function, then characterize the macroscopic elastoplastic behavior of the RFPCs under three-dimensional arbitrary loading/unloading histories.

The local axes of an inclusion are denoted by the primed coordinate system and the fixed or material axes by the unprimed one. With no loss in generality, we let axis $1^{\prime}$ be the symmetric axis of the spheroid and $3^{\prime}$ lie in the 2-3 plane. Denoting $Q_{i j}$ as the directional cosine between the $i$ th primed and $j$ th unprimed axes, we have

$$
x_{i}^{\prime}=\left[Q_{i j}\right] x_{j}
$$


where the transformation matrix has the form of

$$
\left[Q_{i j}\right]=\left[\begin{array}{ccc}
\cos \theta & \sin \theta \cos \phi & \sin \theta \sin \phi \\
-\sin \theta & \cos \theta \cos \phi & \cos \theta \sin \phi \\
0 & -\sin \phi & \cos \phi
\end{array}\right]
$$

in which $\theta$ is the angle between $x_{1}$ and $x_{1}^{\prime}$ and $\phi$ is the angle between $x_{3}$ and $x_{3}^{\prime}$. Note that $\theta$ varies from 0 to $\pi$ and $\phi$ ranges from 0 to $2 \pi$. Any second-rank tensor (e.g., stress tensor) can be transformed as

$$
\sigma_{i j}^{\prime}=Q_{i k} Q_{j l} \sigma_{k l}
$$

For convenience, the governing equations for aligned fiber-reinforced composites given by Ju and Chen (1994a) can be rewritten as (dropping the ensemble averaged brackets)

$$
\begin{aligned}
& \overline{\boldsymbol{\sigma}}=\mathbf{C}_{0}:\left[\overline{\boldsymbol{\epsilon}}-\sum_{r=1}^{n} \phi_{r} \boldsymbol{\epsilon}_{r}^{*}\right] \\
& \overline{\boldsymbol{\epsilon}}=\boldsymbol{\epsilon}^{o}+\sum_{r=1}^{n} \phi_{r} \mathbf{S}: \boldsymbol{\epsilon}_{r}^{*} \\
& \boldsymbol{\epsilon}_{r}^{*}=-\left(\mathbf{S}+\mathbf{A}_{r}\right)^{-1}: \boldsymbol{\epsilon}^{o}
\end{aligned}
$$

where $n$ denotes the number of inclusions of different material properties (excluding the matrix).

When all inclusions are randomly oriented in the three-dimensional space, the composite as a whole is macroscopically isotropic. The symbol $\subset \cdot \supset$ is used to define the orientational averaging process as

$$
\subset \cdot \supset \equiv \int_{0}^{\pi} \int_{0}^{\pi}(\cdot) P(\theta, \phi) \sin \theta d \theta d \phi
$$

where $P(\theta, \phi)$ is the probability density function. In the special case of uniformly random orientation, we have $P(\theta, \phi)=1 / 2 \pi$. Accordingly, after the orientational averaging process, the governing field equations for randomly oriented fiber-reinforced composites can be derived as

$$
\begin{aligned}
& \subset \overline{\boldsymbol{\sigma}} \supset=\mathbf{C}_{0}:\left[\subset \overline{\boldsymbol{\epsilon}} \supset-\sum_{r=1}^{2} \phi_{r} \subset \boldsymbol{\epsilon}_{r}^{*} \supset\right] \\
& \subset \overline{\boldsymbol{\epsilon}} \supset=\boldsymbol{\Upsilon}: \boldsymbol{\epsilon}^{o} \\
& \subset \boldsymbol{\epsilon}_{r}^{*} \supset=-\boldsymbol{\Omega}_{r}: \boldsymbol{\epsilon}^{o}
\end{aligned}
$$

where the fourth-rank tensors $\boldsymbol{\Upsilon}$ and $\boldsymbol{\Omega}_{r}$ read

$$
\begin{aligned}
& \Upsilon_{i j k l}=I_{i j k l}-\sum_{r=1}^{2} \frac{\phi_{r}}{2 \pi} \int_{0}^{\pi} \int_{0}^{\pi} Q_{m i} Q_{n j} S_{m n p q}\left[\left(A_{r}\right)_{p q s t}+S_{p q s t}\right]^{-1} Q_{s k} Q_{t l} \sin \theta d \theta d \phi \\
& \left(\Omega_{r}\right)_{i j k l}=\frac{1}{2 \pi} \int_{0}^{\pi} \int_{0}^{\pi} Q_{m i} Q_{n j}\left[\left(A_{r}\right)_{m n p q}+S_{m n p q}\right]^{-1} Q_{p k} Q_{q l} \sin \theta d \theta d \phi
\end{aligned}
$$


Hence, the effective elastic stiffness tensor $\subset \mathbf{C}_{*} \supset$ for randomly oriented fiber-reinforced composites can be obtained as

$$
\subset \mathbf{C}_{*} \supset=\mathbf{C}_{o} \cdot\left[\mathbf{I}+\sum_{r=1}^{2} \phi_{r} \boldsymbol{\Omega}_{r} \cdot \boldsymbol{\Upsilon}^{-1}\right]
$$

It is noted that the proposed randomly oriented effective stiffness of the composite should be identical to the results based on Mori-Tanaka's method (e.g., Tandon and Weng, 1986).

Before we derive the isotropic elastic constants for RFPCs, we need to consider the following formulas.

For any generalized isotropic fourth-rank tensor, which is expressed as

$$
L_{i j k l}=L_{I K}^{(1)} \delta_{i j} \delta_{k l}+L_{I J}^{(2)}\left(\delta_{i k} \delta_{j l}+\delta_{i l} \delta_{j k}\right)
$$

and satisfying $L_{12}^{(1)}=L_{13}^{(1)}, L_{21}^{(1)}=L_{31}^{(1)}, L_{22}^{(1)}=L_{23}^{(1)}=L_{32}^{(1)}=L_{33}^{(1)}, L_{12}^{(2)}=L_{21}^{(2)}=L_{13}^{(2)}=L_{31}^{(2)}, L_{22}^{(2)}=L_{33}^{(2)}=$ $L_{23}^{(2)}=L_{32}^{(2)}$, the following formulation can be proved after lengthy but straightforward manipulations

$$
\begin{aligned}
\subset L_{i j k l} \supset= & \frac{1}{2 \pi} \int_{0}^{\pi} \int_{0}^{\pi} Q_{m i} Q_{n j} L_{m n p q} Q_{p k} Q_{q l} \sin \theta d \theta d \phi \\
& =v_{1} \delta_{i j} \delta_{k l}+v_{2}\left(\delta_{i k} \delta_{j l}+\delta_{i l} \delta_{j k}\right)
\end{aligned}
$$

where

$$
\begin{aligned}
& v_{1}=\frac{1}{15}\left[L_{11}^{(1)}+4 L_{12}^{(1)}+4 L_{21}^{(1)}+6 L_{22}^{(1)}+2 L_{11}^{(2)}-4 L_{12}^{(2)}+2 L_{22}^{(2)}\right] \\
& v_{2}=\frac{1}{15}\left[L_{11}^{(1)}-L_{12}^{(1)}-L_{21}^{(1)}+L_{22}^{(1)}+2 L_{11}^{(2)}+6 L_{12}^{(2)}+7 L_{22}^{(2)}\right]
\end{aligned}
$$

Similarly, for any transversely isotropic fourth-rank tensor $\tilde{\mathbf{F}}$, which takes form of

$$
M_{i j k l}=\tilde{F}_{i j k l}\left(M_{1}, M_{2}, M_{3}, M_{4}, M_{5}, M_{6}\right)
$$

where the transversely isotropic fourth-rank tensor $\tilde{F}_{i j k l}$ are given Equation 35, the following formulation is obtained.

$$
\begin{aligned}
\subset M_{i j k l} \supset= & \frac{1}{2 \pi} \int_{0}^{\pi} \int_{0}^{\pi} Q_{m i} Q_{n j} M_{m n p q} Q_{p k} Q_{q l} \sin \theta d \theta d \phi \\
& =\zeta_{1} \delta_{i j} \delta_{k l}+\zeta_{2}\left(\delta_{i k} \delta_{j l}+\delta_{i l} \delta_{j k}\right)
\end{aligned}
$$

where

$$
\begin{aligned}
& \zeta_{1}=\frac{1}{15}\left[M_{1}+5\left(M_{3}+M_{4}+3 M_{5}\right)\right] \\
& \zeta_{2}=\frac{1}{15}\left[M_{1}+10 M_{2}+15 M_{6}\right]
\end{aligned}
$$


The formulations in Equations 137 and 141 show that, after the three-dimensional orientational averaging process, any generalized isotropic fourth-rank tensor as well as any transversely isotropic fourth-rank tensor will become the isotropic fourth-rank tensor.

In particular, after the orientational average process, the stiffness tensor for randomly oriented, partially debonded (damaged) fibers can be derived as

$$
\begin{aligned}
\subset \mathbf{C}_{2} \supset= & \frac{1}{2 \pi} \int_{0}^{\pi} \int_{0}^{\pi} Q_{m i} Q_{n j}\left(C_{2}\right)_{m n p q} Q_{p k} Q_{q l} \sin \theta d \theta d \phi \\
& =\chi_{1} \delta_{i j} \delta_{k l}+\chi_{2}\left(\delta_{i k} \delta_{j l}+\delta_{i l} \delta_{j k}\right)
\end{aligned}
$$

with

$$
\begin{aligned}
& \chi_{1}=\frac{1}{15}\left[t_{1}+5\left(t_{3}+t_{4}+3 t_{5}\right)\right] \\
& \chi_{2}=\frac{1}{15}\left[t_{1}+10 t_{2}+15 t_{6}\right]
\end{aligned}
$$

in which the parameters $t_{1}, \ldots t_{6}$ in Equations 145 and 146 are given in Equations 49-54.

With the help of the formulations in Equations 137 and 141, the effective elasticity tensor $\subset \mathbf{C}_{*} \supset$ of RFPCs can be obtained as

$$
\subset \mathbf{C}_{*} \supset=\tilde{c}_{1} \delta_{i j} \delta_{k l}+\tilde{c}_{2}\left(\delta_{i k} \delta_{j l}+\delta_{i l} \delta_{j k}\right)
$$

where

$$
\begin{aligned}
& \tilde{c}_{1}=\frac{1}{15}\left[C_{11}^{(1)}+4 C_{12}^{(1)}+4 C_{21}^{(1)}+6 C_{22}^{(1)}+2 C_{11}^{(2)}-4 C_{12}^{(2)}+2 C_{22}^{(2)}\right] \\
& \tilde{c}_{2}=\frac{1}{15}\left[C_{11}^{(1)}-C_{12}^{(1)}-C_{21}^{(1)}+C_{22}^{(1)}+2 C_{11}^{(2)}+6 C_{12}^{(2)}+7 C_{22}^{(2)}\right]
\end{aligned}
$$

Here, the parameters $C_{11}^{(1)}, \ldots, C_{22}^{(2)}$ are given by Equations 60 and 61 . Altenatively, the effective Young's modulus $E_{*}$ and Poisson's ratio $\nu_{*}$ of RFPCs are easily obtained through the following relations

$$
\begin{aligned}
E_{*} & =\frac{\tilde{c}_{2}\left(3 \tilde{c}_{1}+2 \tilde{c}_{2}\right)}{\tilde{c}_{1}+\tilde{c}_{2}} \\
\nu_{*} & =\frac{\tilde{c}_{1}}{2\left(\tilde{c}_{1}+\tilde{c}_{2}\right)}
\end{aligned}
$$

Similarly, the transforming fourth-rank tensor $\Upsilon$ between macroscopic strain $\subset \overline{\boldsymbol{\epsilon}} \supset$ and far field strain $\epsilon^{o}$ takes the form of

$$
\Upsilon=\Upsilon_{1} \delta_{i j} \delta_{k l}+\Upsilon_{2}\left(\delta_{i k} \delta_{j l}+\delta_{i l} \delta_{j k}\right)
$$

where

$$
\Upsilon_{1}=-\sum_{r=1}^{2} \phi_{r}\left\{\frac{\left[\mathcal{S}_{11}^{(1)}+4 \mathcal{S}_{21}^{(1)}+2 \mathcal{S}_{11}^{(2)}\right]\left[1-\left(\Lambda_{r}\right)_{11}-4\left(\Lambda_{r}\right)_{12}\right]+10 \mathcal{S}_{21}^{(1)}\left(\Lambda_{r}\right)_{12}}{30\left[\left(Z_{r}\right)_{2}+\mathcal{S}_{11}^{(2)}\right]}-\frac{2 \mathcal{S}_{12}^{(2)}}{15\left[\left(Z_{r}\right)_{2}+\mathcal{S}_{12}^{(2)}\right]}\right.
$$




$$
\begin{gathered}
\left.+\frac{\left[3 \mathcal{S}_{22}^{(1)}+2 \mathcal{S}_{12}^{(1)}+3 \mathcal{S}_{22}^{(2)}\right]\left[3-4\left(\Lambda_{r}\right)_{21}-6\left(\Lambda_{r}\right)_{22}\right]-6 \mathcal{S}_{22}^{(2)}+5 \mathcal{S}_{12}^{(1)}\left(\Lambda_{r}\right)_{21}}{45\left[\left(Z_{r}\right)_{2}+\mathcal{S}_{22}^{(2)}\right]}\right\} \\
\Upsilon_{2}=\frac{1}{2}-\sum_{r=1}^{2} \phi_{r}\left\{\frac{\left[\mathcal{S}_{11}^{(1)}-\mathcal{S}_{21}^{(1)}+2 \mathcal{S}_{11}^{(2)}\right]\left[1-\left(\Lambda_{r}\right)_{11}+\left(\Lambda_{r}\right)_{12}\right]}{30\left[\left(Z_{r}\right)_{2}+\mathcal{S}_{11}^{(2)}\right]}+\frac{\mathcal{S}_{12}^{(2)}}{5\left[\left(Z_{r}\right)_{2}+\mathcal{S}_{12}^{(2)}\right]}\right. \\
\left.+\frac{\left[\mathcal{S}_{22}^{(1)}-\mathcal{S}_{12}^{(1)}+\mathcal{S}_{22}^{(2)}\right]\left[1+2\left(\Lambda_{r}\right)_{21}-2\left(\Lambda_{r}\right)_{22}\right]+6 \mathcal{S}_{22}^{(2)}}{30\left[\left(Z_{r}\right)_{2}+\mathcal{S}_{22}^{(2)}\right]}\right\}
\end{gathered}
$$

Similarly, the transformation fourth-rank tensor $\Omega_{r}$ between orientational eigenstrain $\subset \epsilon^{*} \supset$ and far field strain $\epsilon^{o}$ can be computed as

$$
\boldsymbol{\Omega}_{r}=\left(\Omega_{r}\right)_{1} \delta_{i j} \delta_{k l}+\left(\Omega_{r}\right)_{2}\left(\delta_{i k} \delta_{j l}+\delta_{i l} \delta_{j k}\right)
$$

in which

$$
\begin{aligned}
\left(\Omega_{r}\right)_{1} & =\frac{1-\left(\Lambda_{r}\right)_{11}-4\left(\Lambda_{r}\right)_{12}}{30\left[\left(Z_{r}\right)_{2}+S_{11}^{(2)}\right]}-\frac{1}{15\left[\left(Z_{r}\right)_{2}+S_{12}^{(2)}\right]}+\frac{1-4\left(\Lambda_{r}\right)_{21}-6\left(\Lambda_{r}\right)_{22}}{30\left[\left(Z_{r}\right)_{2}+S_{22}^{(2)}\right]} \\
\left(\Omega_{r}\right)_{2} & =\frac{1-\left(\Lambda_{r}\right)_{11}-\left(\Lambda_{r}\right)_{12}}{30\left[\left(Z_{r}\right)_{2}+S_{11}^{(2)}\right]}-\frac{1}{10\left[\left(Z_{r}\right)_{2}+S_{12}^{(2)}\right]}+\frac{7+\left(\Lambda_{r}\right)_{21}-\left(\Lambda_{r}\right)_{22}}{60\left[\left(Z_{r}\right)_{2}+S_{22}^{(2)}\right]}
\end{aligned}
$$

Assuming all reinforcements of the composites changed from aligned array to randomly oriented array in the three-dimensional space, by using the orientational averaging process, the orientation-averaged square of stress norm $\subset H_{m} \supset$ can be obtained as

$$
\subset H_{m} \supset=\boldsymbol{\sigma}^{o}: \subset \mathbf{T} \supset: \boldsymbol{\sigma}^{o}
$$

where the isotropic fourth-rank tensor $\subset \mathbf{T} \supset$ is

$$
\subset T_{i j k l} \supset=\frac{1}{2 \pi} \int_{0}^{\pi} \int_{0}^{\pi} Q_{m i} Q_{n j} T_{m n p q} Q_{p k} Q_{q l} \sin \theta d \theta d \phi
$$

The components of the positive definite fourth-rank tensor $\subset \mathbf{T} \supset$ read

$$
\subset T_{i j k l} \supset=T_{1} \delta_{i j} \delta_{k l}+T_{2}\left(\delta_{i k} \delta_{j l}+\delta_{i l} \delta_{j k}\right)
$$

with

$$
\begin{aligned}
& T_{1}=\frac{1}{15}\left[T_{11}^{(1)}+4 T_{12}^{(1)}+4 T_{21}^{(1)}+6 T_{22}^{(1)}+2 T_{11}^{(2)}-4 T_{12}^{(2)}+2 T_{22}^{(2)}\right] \\
& T_{2}=\frac{1}{15}\left[T_{11}^{(1)}-T_{12}^{(1)}-T_{21}^{(1)}+2 T_{11}^{(2)}+6 T_{12}^{(2)}+7 T_{22}^{(2)}\right]
\end{aligned}
$$

In Equation 158, $\subset H_{m} \supset$ is described in terms of the far-field satress $\boldsymbol{\sigma}^{\circ}$. Alternatively, the orientationaveraged square of the stress norm can also be expressed in terms of the macroscopic (orientation- averaged) 
stress $\overline{\boldsymbol{\sigma}}$. Following Ju and Chen (1994a), the relationship between the far-field stress $\boldsymbol{\sigma}^{\circ}$ and the macroscopic stress $\overline{\boldsymbol{\sigma}}$ takes the form

$$
\boldsymbol{\sigma}^{o}=\subset \mathbf{P} \supset: \overline{\boldsymbol{\sigma}}
$$

where the components of $\subset \mathbf{P} \supset$ are

$$
\subset \mathbf{P} \supset=\subset P_{1} \supset \delta_{i j} \delta_{k l}+\subset P_{2} \supset\left(\delta_{i k} \delta_{j l}+\delta_{i l} \delta_{j k}\right)
$$

with

$$
\begin{aligned}
& \subset P_{1} \supset=\sum_{r=1}^{2}\left\{\frac{\phi_{r}\left[\Upsilon_{1}-\left(\Omega_{r}\right)_{1}\right]}{\left\{1+2 \phi_{r}\left[\left(\Omega_{r}\right)_{2}-\Upsilon_{2}\right]\right\}\left\{1+\phi_{r}\left[3\left(\Omega_{r}\right)_{1}+2\left(\Omega_{r}\right)_{2}-3 \Upsilon_{1}-2 \Upsilon_{2}\right]\right\}}\right\} \\
& \subset P_{2} \supset=\sum_{r=1}^{2}\left\{\frac{1}{2+4 \phi_{r}\left[\left(\Omega_{r}\right)_{2}-\Upsilon_{2}\right]}\right\}
\end{aligned}
$$

By combining Equations 158 and 163, we arrive at the alternative expression for the orientation-averaged current stress norm (square) in a matrix point

$$
\subset H_{m} \supset=\overline{\boldsymbol{\sigma}}: \subset \overline{\mathbf{T}} \supset: \overline{\boldsymbol{\sigma}}
$$

where

$$
\begin{aligned}
\subset \overline{\mathbf{T}} \supset & =\subset \overline{\mathbf{P}} \supset \cdot \subset \mathbf{T} \supset \cdot \subset \overline{\mathbf{P}} \supset \\
& =\bar{T}_{1} \delta_{i j} \delta_{k l}+\bar{T}_{2}\left(\delta_{i k} \delta_{j l}+\delta_{i l} \delta_{j k}\right)
\end{aligned}
$$

with

$$
\begin{aligned}
& \bar{T}_{1}=\left[3 \subset P_{1} \supset+2 \subset P_{2} \supset\right]^{2} T_{1}+2 \subset P_{1} \supset T_{2}\left[3 \subset P_{1} \supset+4 \subset P_{2} \supset\right] \\
& \bar{T}_{2}=4 \subset P_{2} \supset^{2} T_{2}
\end{aligned}
$$

Similar to Section 3.2, the effective yield function for the three-phase RFPCs can be proposed as

$$
\subset \bar{F} \supset=\left(1-\phi_{1}\right)^{2} \overline{\boldsymbol{\sigma}}: \subset \overline{\mathbf{T}} \supset: \overline{\boldsymbol{\sigma}}-K^{2}\left(\bar{e}^{p}\right)
$$

with the isotropic hardening function $K\left(\bar{e}^{p}\right)$ for the three-phase composite. It should be noted that the effective yield function is pressure dependent and not of the von Mises type. Moreover, for simplicity, we assume that the overall flow rule for the matrix is associative. Therefore, the effective ensemble-volume, averaged plastic strain rate for the composite can be expressed as

$$
\dot{\overline{\boldsymbol{\epsilon}}}^{p}=\dot{\lambda} \frac{\partial \subset \bar{F} \supset}{\partial \overline{\boldsymbol{\sigma}}}=2\left(1-\phi_{1}\right)^{2} \dot{\lambda} \subset \overline{\mathbf{T}} \supset: \overline{\boldsymbol{\sigma}}
$$


where $\dot{\lambda}$ denotes the plastic consistency parameter.

Inspired by the structure of the micromechanically derived stress norm, the effective equivalent plastic strain rate for the composite is defined as

$$
\dot{\bar{e}}^{p} \equiv \sqrt{\frac{2}{3} \dot{\bar{\epsilon}}^{p}: \subset \overline{\mathbf{T}} \supset^{-1}: \dot{\overline{\boldsymbol{\epsilon}}}^{p}}=2\left(1-\phi_{1}\right)^{2} \dot{\lambda} \sqrt{\frac{2}{3} \overline{\boldsymbol{\sigma}}: \subset \overline{\mathbf{T}} \supset: \overline{\boldsymbol{\sigma}}}
$$

The $\dot{\lambda}$ together with the yield function $\bar{F}$ must obey the Kuhn-Tucker loading/unloading conditions and consistency requrements

$$
\begin{gathered}
\dot{\lambda} \geq 0, \subset \bar{F} \supset \leq 0, \dot{\lambda} \subset \bar{F} \supset=0 \\
\dot{\lambda} \subset \dot{\bar{F}} \supset=0
\end{gathered}
$$

It is emphasized that the ensemble-volume averaged yield function in Equation 171, the averaged plastic flow rule in Equation 172, the equivalent plastic strain rate in Equation 173, and the Kuhn-Tucker conditions completely characterize the effective plasticity formulation for a composite material with any isotropic hardening function $K\left(\bar{e}^{p}\right)$. It is acceptible to extend the proposed model to accommodate kinematic hardening. In what follows, the simple power-law type isotropic hardening function is employed as an example

$$
K\left(\bar{e}^{p}\right)=\sqrt{\frac{2}{3}}\left\{\sigma_{y}+h\left(\bar{e}^{p}\right)^{\bar{q}}\right\}
$$

where $\sigma_{y}$ is the initial yield stress, and $h$ and $\bar{q}$ signify the linear and exponential isotropic hardening parameters, respectively, for the three-phase composite.

\subsection{ELASTOPLASTIC STRESS-STRAIN RELATIONS FOR RFPCs}

In order to illustrate the proposed micromechanics-based elastoplastic damage model for RFPCs, we consider the examples of the uniaxial, biaxial, and triaxial tensile loadings in the following.

\subsubsection{Uniaxial Elastoplastic Stress-strain Relation}

The applied macroscopic stress $\overline{\boldsymbol{\sigma}}$ can be written as

$$
\bar{\sigma}_{11} \neq 0, \quad \text { all other } \bar{\sigma}_{i j}=0
$$

With the isotropic hardening law described by Equation 176, the overall yield function becomes

$$
\subset \bar{F}\left(\overline{\boldsymbol{\sigma}}, \bar{e}^{p}\right) \supset=\left(1-\phi_{1}\right)^{2} \overline{\boldsymbol{\sigma}}: \subset \overline{\mathbf{T}} \supset: \overline{\boldsymbol{\sigma}}-\frac{2}{3}\left\{\sigma_{y}+h\left(\bar{e}^{p}\right)^{\bar{q}}\right\}^{2}
$$


Therefore, the effective yield function for the uniaxial tensile loading reads

$$
\subset \bar{F} \supset=\left(1-\phi_{1}\right)^{2}\left(\subset \bar{T}_{1} \supset+2 \subset \bar{T}_{2} \supset\right) \bar{\sigma}_{11}^{2}-\frac{2}{3}\left\{\sigma_{y}+h\left(\bar{e}^{p}\right)^{\bar{q}}\right\}^{2}
$$

The macroscopic incremental plastic strain rate defined by Equation 172 becomes

$$
\triangle \overline{\boldsymbol{\epsilon}}^{p}=2\left(1-\phi_{1}\right)^{2} \triangle \lambda \bar{\sigma}_{11}\left(\begin{array}{ccc}
\subset \bar{T}_{1} \supset+2 \subset \bar{T}_{2} \supset & 0 & 0 \\
0 & \subset \bar{T}_{1} \supset & 0 \\
0 & 0 & \subset \bar{T}_{1} \supset
\end{array}\right)
$$

for any stress beyond the initial yielding. Similarly, the incremental equivalent plastic strain can be expressed as

$$
\triangle \bar{e}^{p}=2\left(1-\phi_{1}\right)^{2} \triangle \lambda\left|\bar{\sigma}_{11}\right| \sqrt{\frac{2}{3}\left(\subset \bar{T}_{1} \supset+2 \subset \bar{T}_{2} \supset\right)}
$$

From the linear elasticity, the macroscopic incremental elastic strain reads

$$
\triangle \overline{\boldsymbol{\epsilon}}^{e}=\left(\begin{array}{ccc}
1 & 0 & 0 \\
0 & -\nu_{*} & 0 \\
0 & 0 & -\nu_{*}
\end{array}\right) \frac{\triangle \bar{\sigma}_{11}}{E_{*}}
$$

In addition, as given in Equation 73, the total incremental strain is the sum of the elastic incremental strain and plastic incremental strain.

The positive parameter $\lambda=\sum_{i}(\Delta \lambda)^{i}$ is solved from the nonlinear equation obtained by enforcing the plastic consistency condition $\subset \bar{F} \supset=0$. Since only the uniaxial loading is under consideration, the nonlinear equation takes the form (cf. Equation 9)

$$
\left(1-\phi_{1}\right)^{2}\left(\subset \bar{T}_{1} \supset+2 \subset \bar{T}_{2} \supset\right) \bar{\sigma}_{11}^{2}=\frac{2}{3}\left\{\sigma_{y}+h\left[2\left(1-\phi_{1}\right)^{2} \lambda \sqrt{\frac{2}{3}\left(\subset \bar{T}_{1} \supset+2 \subset \bar{T}_{2} \supset\right)}\left|\bar{\sigma}_{11}\right|\right]^{\bar{q}}\right\}^{2}
$$

Specifically, for a monotonic uniaxial loading, the overall uniaxial stress-strain relation can be derived by integrating Equations 180 and 182:

$$
\overline{\boldsymbol{\epsilon}}=\left(\begin{array}{ccc}
1 & 0 & 0 \\
0 & -\nu_{*} & 0 \\
0 & 0 & -\nu_{*}
\end{array}\right) \frac{\bar{\sigma}_{11}}{E_{*}}+2\left(1-\phi_{1}\right)^{2} \sum_{i}\left[(\triangle \lambda)^{i}\left(\bar{\sigma}_{11}\right)^{i}\right]\left(\begin{array}{ccc}
\subset \bar{T}_{1} \supset+2 \subset \bar{T}_{2} \supset & 0 & 0 \\
0 & \subset \bar{T}_{1} \supset & 0 \\
0 & 0 & \subset \bar{T}_{1} \supset
\end{array}\right)
$$

where $E_{*}$ and $\nu_{*}$ are given Equations 150and 151. In addition, $(\triangle \lambda)^{i}$ is the $i$-th iteration value of $\triangle \lambda$, and $\left(\bar{\sigma}_{11}\right)^{i}$ is the $i$-th iteration value of macroscopic uniaxial stress. 


\subsubsection{Biaxial Elastoplastic Stress-strain Relation}

The applied macroscopic stress $\overline{\boldsymbol{\sigma}}$ can be rephrased as

$$
\bar{\sigma}_{22}=\bar{\sigma}_{33} \neq 0, \quad \text { all other } \bar{\sigma}_{i j}=0 .
$$

Substituting Equation 185 into Equation 178, the effective yield function for the case of biaxial loading becomes

$$
\subset \bar{F} \supset=4\left(1-\phi_{1}\right)^{2}\left(\subset \bar{T}_{1} \supset+\subset \bar{T}_{2} \supset\right) \bar{\sigma}_{22}^{2}-\frac{2}{3}\left\{\sigma_{y}+h\left(\bar{e}^{p}\right)^{\bar{q}}\right\}^{2}
$$

The macroscopic incremental plastic strain rate defined by Equation 172 takes the form

$$
\triangle \overline{\boldsymbol{\epsilon}}^{p}=4\left(1-\phi_{1}\right)^{2} \triangle \lambda \bar{\sigma}_{22}\left(\begin{array}{ccc}
\subset \bar{T}_{1} \supset & 0 & 0 \\
0 & \subset \bar{T}_{1} \supset+\subset \bar{T}_{2} \supset & 0 \\
0 & 0 & \subset \bar{T}_{1} \supset+\subset \bar{T}_{2} \supset
\end{array}\right)
$$

for any stress beyond the initial yielding. Further, the incremental equivalent plastic strain can be recast as

$$
\triangle \bar{e}^{p}=4\left(1-\phi_{1}\right)^{2} \triangle \lambda\left|\bar{\sigma}_{22}\right| \sqrt{\frac{2}{3}\left(\subset \bar{T}_{1} \supset+\subset \bar{T}_{2} \supset\right)}
$$

The macroscopic incremental elastic strain is

$$
\triangle \overline{\boldsymbol{\epsilon}}^{e}=\left(\begin{array}{ccc}
-2 \nu_{*} & 0 & 0 \\
0 & 1-\nu_{*} & 0 \\
0 & 0 & 1-\nu_{*}
\end{array}\right) \frac{\triangle \bar{\sigma}_{22}}{E_{*}}
$$

The positive parameter $\lambda=\sum_{i}(\triangle \lambda)^{i}$ is solved from the plastic consistency condition $\subset \bar{F} \supset=0$. For the case of biaxial loading, the nonlinear equation arrives at (cf. Equation 34)

$$
4\left(1-\phi_{1}\right)^{2}\left(\subset \bar{T}_{1} \supset+\subset \bar{T}_{2} \supset\right) \bar{\sigma}_{22}^{2}=\frac{2}{3}\left\{\sigma_{y}+h\left[4\left(1-\phi_{1}\right)^{2} \lambda \sqrt{\frac{2}{3}\left(\subset \bar{T}_{1} \supset+\subset \bar{T}_{2} \supset\right)}\left|\bar{\sigma}_{22}\right|\right]^{\bar{q}}\right\}^{2}
$$

In the special case of a monotonic biaxial loading, the overall biaxial stress-strain relation can be obtained by integrating Equations 187 and 189:

$$
\overline{\boldsymbol{\epsilon}}=\left(\begin{array}{ccc}
-2 \nu_{*} & 0 & 0 \\
0 & 1-\nu_{*} & 0 \\
0 & 0 & 1-\nu_{*}
\end{array}\right) \frac{\bar{\sigma}_{22}}{E_{*}}+
$$




$$
4\left(1-\phi_{1}\right)^{2} \sum_{i}\left[(\triangle \lambda)^{i}\left(\bar{\sigma}_{22}\right)^{i}\right]\left(\begin{array}{ccc}
\subset \bar{T}_{1} \supset & 0 & 0 \\
0 & \subset \bar{T}_{1} \supset+\subset \bar{T}_{2} \supset & 0 \\
0 & 0 & \subset \bar{T}_{1} \supset+\subset \bar{T}_{2} \supset
\end{array}\right)
$$

where $(\triangle \lambda)^{i}$ is the $i$-th iteration value of $\triangle \lambda$, and $\left(\bar{\sigma}_{22}\right)^{i}$ is the $i$-th iteration value of macroscopic biaxial stress.

\subsubsection{Triaxial Elastoplastic Stress-strain Relation}

The applied macroscopic stress $\overline{\boldsymbol{\sigma}}$ can be written as

$$
\bar{\sigma}_{22}=\bar{\sigma}_{33}=\iota \bar{\sigma}_{11} \neq 0, \quad \text { all other } \bar{\sigma}_{i j}=0 .
$$

where $\iota$ is the proportional loading ratio. In particular, $\iota=1$ indicates a pure hydrostatic loading. Substituting Equation 192into Equation 178, the effective yield function for the case of triaxial loading is written as

$$
\left.\subset \bar{F} \supset=\left(1-\phi_{1}\right)^{2}\left[(2 \iota+1)^{2} \subset \bar{T}_{1} \supset+2\left(2 \iota^{2}+1\right) \subset \bar{T}_{2} \supset\right)\right] \bar{\sigma}_{11}^{2}-\frac{2}{3}\left\{\sigma_{y}+h\left(\bar{e}^{p}\right)^{\bar{q}}\right\}^{2}
$$

The macroscopic incremental plastic strain rate defined by Equation 172 becomes

$$
\begin{aligned}
& \triangle \overline{\boldsymbol{\epsilon}}^{p}=2\left(1-\phi_{1}\right)^{2} \triangle \lambda \bar{\sigma}_{11} * \\
& \left(\begin{array}{ccc}
(2 \iota+1) \subset \bar{T}_{1} \supset+2 \subset \bar{T}_{2} \supset & 0 \\
0 & (2 \iota+1) \subset \bar{T}_{1} \supset+2 \iota \subset \bar{T}_{2} \supset & 0 \\
0 & 0 & (2 \iota+1) \subset \bar{T}_{1} \supset+2 \iota \subset \bar{T}_{2} \supset
\end{array}\right)
\end{aligned}
$$

for any stress beyond the initial yielding. Moreover, the incremental equivalent plastic strain can be expressed as

$$
\triangle \bar{e}^{p}=2\left(1-\phi_{1}\right)^{2} \triangle \lambda\left|\bar{\sigma}_{11}\right| \sqrt{\frac{2}{3}\left[(2 \iota+1)^{2} \subset \bar{T}_{1} \supset+2\left(2 \iota^{2}+1\right) \subset \bar{T}_{2} \supset\right]}
$$

The macroscopic incremental elastic strain reads

$$
\triangle \overline{\boldsymbol{\epsilon}}^{e}=\left(\begin{array}{ccc}
1-2 \iota \nu_{*} & 0 & 0 \\
0 & \iota-(1+\iota) \nu_{*} & 0 \\
0 & 0 & \iota-(1+\iota) \nu_{*}
\end{array}\right) \frac{\triangle \bar{\sigma}_{11}}{E_{*}}
$$

Similarly, the positive parameter $\lambda=\sum_{i}(\triangle \lambda)^{i}$ is solved by enforcing the plastic consistency condition $\subset \bar{F} \supset=0$ as follows (cf. Equation 195):

$$
\left(1-\phi_{1}\right)^{2}\left[(2 \iota+1)^{2} \subset \bar{T}_{1} \supset+2\left(2 \iota^{2}+1\right) \subset \bar{T}_{2} \supset\right] \sigma_{11}^{2}
$$




$$
=\frac{2}{3}\left\{\sigma_{y}+h\left[2\left(1-\phi_{1}\right)^{2} \lambda \sqrt{\frac{2}{3}\left[(2 \iota+1)^{2} \subset \bar{T}_{1} \supset+2\left(2 \iota^{2}+1\right) \subset \bar{T}_{2} \supset\right]}\left|\bar{\sigma}_{11}\right|\right]^{\bar{q}}\right\}^{2}
$$

In the special case of a monotonic triaxial loading, the overall triaxial stress-strain relation can be derived by integrating Equations 194 and 196:

$$
\begin{aligned}
& \overline{\boldsymbol{\epsilon}}=\left(\begin{array}{ccc}
1-2 \iota \nu_{*} & 0 & 0 \\
0 & \iota-(1+\iota) \nu_{*} & 0 \\
0 & 0 & \iota-(1+\iota) \nu_{*}
\end{array}\right) \frac{\bar{\sigma}_{11}}{E_{*}}+2\left(1-\phi_{1}\right)^{2} \sum_{i}\left[(\triangle \lambda)^{i}\left(\bar{\sigma}_{11}\right)^{i}\right] * \\
& \left(\begin{array}{ccc}
(2 \iota+1) \subset \bar{T}_{1} \supset+2 \subset \bar{T}_{2} \supset & 0 & 0 \\
0 & (2 \iota+1) \subset \bar{T}_{1} \supset+2 \iota \subset \bar{T}_{2} \supset & 0 \\
0 & 0 & (2 \iota+1) \subset \bar{T}_{1} \supset+2 \iota \subset \bar{T}_{2} \supset
\end{array}\right)
\end{aligned}
$$

where $(\Delta \lambda)^{i}$ is the $i$-th iteration value of $\triangle \lambda$, and $\left(\bar{\sigma}_{11}\right)^{i}$ is the $i$-th iteration value of macroscopic triaxial stress. 


\section{FAILURE AND DAMAGE EVOLUTION}

\subsection{FAILURE IN DISCONTONUOUS FIBER COMPOSITES}

Failure modes in discontinuous fiber composites (which have a complex structure) are best understood in terms of the arrangement of reinforcing fibers and matrix resin. The dominating failure mechanisms in the composite structures take place under tensile and compressive states; therefore, failure modes are mainly classified into tensile failure mode and compressive failure mode.

Tensile failure usually occurs with some separation of fibers along the parallel planes, but the separation is not very clear. Therefore, it is difficult to obtain simple photomicrographs of typical failures. Failure in these chopped-fiber composite materials has resulted in complete debonding between fibers and matrix, although matrix residue is visible on many of the fibers. Compressive failures of discontinuous fiber composites are often similar to tensile failures in that separation between the fiber planes occurs. For a compressive stress in the plane of the sheet, failure begins with delamination, followed quickly by buckling of the resulting thin layers. Thus, this type of failure is characterized by the sharp kinking of the fibers, with occasional nonkinked protruding filament. Accordingly, each failure mode indicating the loss of the tensile and/or compressive strength of the composite is quite different. They are most critical for the dissipation of kinetic energy in the mechanical model. We refer to Robertson and Mindroiu (1987) and Matzenmiller and Schweizerhof (1991) for literature review.

The different failure mechanisms of laminate composites due to tension and compression, caused by stresses in fiber direction or perpendicular to the reinforcement, have been cast into failure criteria with experimental evidence by a number of researchers (Hashin, 1980; Hahn et al., 1982; Christensen and Swanson, 1988). However, new failure criteria based on experimental verifications are needed for performing failure analysis of RFPCs, since the failure mechanisms of discontinuous fiber composites are different from those for laminates.

Meanwhile, micromechanical approaches enable us to evaluate and predict local stress and strain fields in each constituent. In addition, these approaches allow us to address the local fluctuations due to the onset and the evolution of damage mechanisms. Therefore, the direct calculation of failure loads and failure behavior on the basis of micromechanical models is essential, even though it is complex.

We consider the interfacial debonding that was proposed as the important failure mechanism in discontinuous fiber composites based on experimental results. 


\subsection{EVOLUTIONARY INTERFACIAL DEBONDING: PROBABILISTIC MICROMECHAN- ICS}

Progressive interfacial debonding may occur under increasing deformations and influence the overall stress-strain behavior of randomly oriented, discontinuous fiber reinforced composites. After the interfacial debonding between fibers and the matrix, the debonded fibers lose the load-carrying capacity in the debonded direction and are regarded as partially debonded fibers. Within the context of the first-order (noninteracting) approximation, the stresses inside fibers should be uniform. For convenience, following Tohgo and Weng (1994) and Zhao and Weng $(1995,1996,1997)$, we employ the average internal stresses of fibers as the controlling factor. The probability of partial debonding is modeled as a two-parameter Weibull process (see Tohgo and Weng [1994], Zhao and Weng [1995], and Ju and Lee [1999]). Assuming that the Weibull (1951) statistics governs, we can express the cumulative probability distribution function of fiber debonding (damage), $P_{d}$, at the level of hydrostatic tensile stress $\left(\overline{\boldsymbol{\sigma}}_{m}\right)_{1}$ as

$$
P_{d}\left[\left(\overline{\boldsymbol{\sigma}}_{m}\right)_{1}\right]=1-\exp \left[-\left(\frac{\left(\overline{\boldsymbol{\sigma}}_{m}\right)_{1}}{S_{o}}\right)^{M}\right]
$$

where $\left(\overline{\boldsymbol{\sigma}}_{m}\right)_{1}=\left[\left(\sigma_{11}\right)_{1}+\left(\sigma_{22}\right)_{1}+\left(\sigma_{33}\right)_{1}\right] / 3$ is the hydrostatic tensile stress of the fibers, the subscript $(\cdot)_{1}$ denotes the fiber phase, and $S_{o}$ and $M$ are the Weibull parameters.

With this function, the average interfacial strength of the interface is related to the Weibull parameters through the Gamma function $\Gamma(\cdot)$ as

$$
\overline{\boldsymbol{\sigma}}_{s}^{p}=S_{o} \Gamma\left(1+\frac{1}{M}\right)
$$

where

$$
\Gamma(b)=\int_{0}^{\infty} y^{b-1} \exp (-y) d y
$$

Therefore, the current partially debonded fiber volume fraction $\phi_{2}$ at a given level of $\left(\overline{\boldsymbol{\sigma}}_{m}\right)_{1}$ is given by

$$
\phi_{2}=\phi P_{d}\left[\left(\overline{\boldsymbol{\sigma}}_{m}\right)_{1}\right]=\phi\left\{1-\exp \left[-\left(\frac{\left(\overline{\boldsymbol{\sigma}}_{m}\right)_{1}}{S_{o}}\right)^{M}\right]\right\}
$$

where $\phi$ is the original fiber volume fraction.

Next we derive the internal stresses of fibers required for the initiation of interfacial debonding. According to the Eshelby's equivalence principle, the perturbed strain field $\boldsymbol{\epsilon}^{\prime}(\mathbf{x})$ induced by inhomogeneities can be related to specified eigenstrains $\epsilon^{*}(\mathbf{x})$ by replacing the inhomogeneities with the matrix material. That is, for the domain of the $q$-phase with elastic stiffness tensor $\mathbf{C}_{q}$, we have

$$
\boldsymbol{\sigma}_{q}=\mathbf{C}_{q}:\left[\boldsymbol{\epsilon}^{o}+\boldsymbol{\epsilon}^{\prime}(\mathbf{x})\right]=\mathbf{C}_{0}:\left[\boldsymbol{\epsilon}^{o}+\boldsymbol{\epsilon}^{\prime}(\mathbf{x})-\boldsymbol{\epsilon}^{*}(\mathbf{x})\right]
$$


where $\boldsymbol{\sigma}_{q}$ is the total local stress for the $q$-phase.

The ensemble-volume averaged strain for three-phase composites takes the form (e.g., Ju and Chen, 1994b):

$$
\overline{\boldsymbol{\epsilon}}=\boldsymbol{\epsilon}^{o}+\sum_{r=1}^{2} \phi_{r} \mathbf{S}: \overline{\boldsymbol{\epsilon}}_{r}^{*}
$$

where the "noninteracting" solution $\boldsymbol{\epsilon}_{q}^{* o}$ of the eigenstrain $\boldsymbol{\epsilon}_{q}^{*}$ has previously been given in Equation 84 . Substituting Equation 84 into 204, we arrive at

$$
\overline{\boldsymbol{\epsilon}}=\left[\mathbf{I}-\sum_{m=1}^{2} \phi_{m} \mathbf{S} \cdot\left(\mathbf{A}_{m}+\mathbf{S}\right)^{-1}\right]: \boldsymbol{\epsilon}^{o}
$$

The volume-averaged stress tensor for $q$-phase is defined as

$$
\overline{\boldsymbol{\sigma}}_{q} \equiv \frac{1}{V_{q}} \int_{V_{q}} \boldsymbol{\sigma}_{q}(\mathbf{x}) d \mathbf{x}=\frac{1}{V_{q}}\left\{\int_{V_{q}} \mathbf{C}_{q}:\left[\boldsymbol{\epsilon}^{o}+\boldsymbol{\epsilon}^{\prime}(\mathbf{x})\right] d \mathbf{x}\right\} \equiv \mathbf{C}_{q}:\left[\boldsymbol{\epsilon}^{o}+\overline{\boldsymbol{\epsilon}}_{q}^{\prime}\right]
$$

where $\overline{\boldsymbol{\epsilon}}_{q}^{\prime}$ can be recast as

$$
\overline{\boldsymbol{\epsilon}}_{q}^{\prime}=\mathbf{S}:\left[\frac{1}{V_{q}} \int_{V_{q}} \overline{\boldsymbol{\epsilon}}^{*}\left(\mathbf{x}^{\prime}\right) d \mathbf{x}^{\prime}\right]=\mathbf{S}: \overline{\boldsymbol{\epsilon}}_{q}^{*}
$$

By combining Equations 84 and 205-207, we arrive at

$$
\overline{\boldsymbol{\sigma}}_{q}=\mathbf{C}_{q} \cdot\left[\mathbf{I}-\mathbf{S} \cdot\left(\mathbf{A}_{q}+\mathbf{S}\right)^{-1}\right] \cdot\left[\mathbf{I}-\sum_{m=1}^{2} \phi_{m} \mathbf{S} \cdot\left(\mathbf{A}_{m}+\mathbf{S}\right)^{-1}\right]^{-1}: \overline{\boldsymbol{\epsilon}}
$$

The above equation is equally applicable to ensemble-volume averaged quantities by applying the ensemble averaging operator $\langle\cdot\rangle$ to $\overline{\boldsymbol{\sigma}}_{q}$ and $\overline{\boldsymbol{\epsilon}}$. Therefore, the averaged internal stresses of fibers can be expressed as $(q=1)$

$$
\overline{\boldsymbol{\sigma}}_{1}=\mathbf{C}_{1} \cdot\left[\mathbf{I}-\mathbf{S} \cdot\left(\mathbf{A}_{1}+\mathbf{S}\right)^{-1}\right] \cdot\left[\mathbf{I}-\sum_{m=1}^{2} \phi_{m} \mathbf{S} \cdot\left(\mathbf{A}_{m}+\mathbf{S}\right)^{-1}\right]^{-1}: \overline{\boldsymbol{\epsilon}} \equiv \mathbf{U}: \overline{\boldsymbol{\epsilon}}
$$

By carrying out lengthy algebra, the components of the positive definite fourth-rank tensor $\mathbf{U}$ are explicitly given by

$$
U_{i j k l}=\tilde{F}_{i j k l}\left(U_{1}, U_{2}, U_{3}, U_{4}, U_{5}, U_{6}\right)
$$

where the definition of fourth-rank tensor $\tilde{\mathbf{F}}$ is given Equation 35 and the inverse and product of fourth-rank tensor $\tilde{\mathbf{F}}$ are given in the Appendix of Ju and Chen (1994b). The components of the fourth-rank tensor $\mathbf{U}$ are given by

$$
\begin{aligned}
& U_{1}=2 \mu_{1} \mathcal{A}_{1} \\
& U_{2}=2 \mu_{1} \mathcal{A}_{2} \\
& U_{3}=\lambda_{1}\left(\mathcal{A}_{1}+4 \mathcal{A}_{2}+3 \mathcal{A}_{3}\right)+2 \mu_{1} \mathcal{A}_{3}
\end{aligned}
$$




$$
\begin{aligned}
& U_{4}=2 \mu_{1} \mathcal{A}_{4} \\
& U_{5}=\lambda_{1}\left(\mathcal{A}_{4}+3 \mathcal{A}_{5}+2 \mathcal{A}_{6}\right)+2 \mu_{1} \mathcal{A}_{5} \\
& U_{6}=2 \mu_{1} \mathcal{A}_{6}
\end{aligned}
$$

where $\mathcal{A}_{1}, \ldots, \mathcal{A}_{6}$ are the parameters of the fourth-rank tensor $\tilde{F}_{i j k l}\left(\mathcal{A}_{1}, \ldots, \mathcal{A}_{6}\right)$, which is the product between two fourth-rank tensors $\tilde{F}_{i j k l}\left(j_{1}, \ldots, j_{6}\right)$ and $\tilde{F}_{i j k l}\left(i_{1}, \ldots, i_{6}\right)$. Parameters $j_{1}, \ldots, j_{6}$ are defined as

$$
\begin{aligned}
& j_{1}=-S_{1}\left(b_{1}+4 b_{2}+b_{3}+2 b_{6}\right)-4 S_{2}\left(b_{1}+2 b_{2}+b_{3}\right)-S_{4}\left(b_{1}+4 b_{2}+3 b_{3}\right)-2 S_{6} b_{1} \\
& j_{2}=-2 S_{2}\left(b_{2}+b_{6}\right)-2 S_{6} b_{2} \\
& j_{3}=-S_{3}\left(b_{1}+4 b_{2}+b_{3}+2 b_{6}\right)-S_{5}\left(b_{1}+4 b_{2}+3 b_{3}\right)-2 S_{6} b_{3} \\
& j_{4}=-S_{1}\left(b_{4}+b_{5}\right)-4 S_{2}\left(b_{4}+b_{5}\right)-S_{4}\left(b_{4}+3 b_{5}+2 b_{6}\right)-2 S_{6} b_{4} \\
& j_{5}=-S_{3}\left(b_{4}+b_{5}\right)-S_{5}\left(b_{4}+3 b_{5}+2 b_{6}\right)-2 S_{6} b_{5} \\
& j_{6}=\frac{1}{2}-2 S_{6} b_{6}
\end{aligned}
$$

in which the components of Eshelby's tensor for a spheroidal inclusion, $S_{1}, \ldots, S_{6}$, are given in Equations $36-41$ and $b_{1}, \ldots, b_{6}$ are the parameters of the fourth-rank tensor $\tilde{F}_{i j k l}\left(b_{1}, \ldots, b_{6}\right)$, which is the inverse of $\tilde{F}_{i j k l}\left(d_{1}, \ldots, d_{6}\right)$ with the following parameters

$$
\begin{aligned}
& d_{1}=S_{1}, d_{2}=S_{2}, d_{3}=S_{3}, \\
& d_{4}=S_{4}, d_{5}=\frac{1}{3}\left(\frac{\kappa_{0}}{\kappa_{1}-\kappa_{0}}-\frac{\mu_{0}}{\mu_{1}-\mu_{0}}\right)+S_{5}, d_{6}=\frac{1}{2} \frac{\mu_{0}}{\mu_{1}-\mu_{0}}+S_{6}
\end{aligned}
$$

In addition, $i_{1}, \ldots, i_{6}$ are the parameters of the fourth-rank tensor $\tilde{F}_{i j k l}\left(i_{1}, \ldots, i_{6}\right)$, which is the inverse of $\tilde{F}_{i j k l}\left(h_{1}, \ldots, h_{6}\right)$ with the following parameters

$$
\begin{aligned}
& h_{1}=\phi_{1} c_{1}-\phi_{2} g_{1}, h_{2}=-\phi_{1} c_{2}-\phi_{2} g_{2}, h_{3}=-\phi_{1} c_{3}-\phi_{2} g_{3} \\
& h_{4}=-\phi_{1} c_{4}-\phi_{2} g_{4}, h_{5}=-\phi_{1} c_{5}-\phi_{2} g_{5}, h_{6}=\frac{1}{2}-\phi_{1} c_{6}-\phi_{2} g_{6}
\end{aligned}
$$

where $c_{1}, \ldots, c_{6}$ are the parameters of the fourth-rank tensor $\tilde{F}_{i j k l}\left(c_{1}, \ldots, c_{6}\right)$, which is the product between two fourth-rank tensors $\tilde{F}_{i j k l}\left(S_{1}, \ldots, S_{6}\right)$ and $\tilde{F}_{i j k l}\left(b_{1}, \ldots, b_{6}\right)$. Furthermore, $g_{1}, \ldots, g_{6}$ are the parameters of the fourth-rank tensor $\tilde{F}_{i j k l}\left(g_{1}, \ldots, g_{6}\right)$, which is the product between two fourth-rank tensors $\tilde{F}_{i j k l}\left(S_{1}, \ldots, S_{6}\right)$ and $\tilde{F}_{i j k l}\left(e_{1}, \ldots, e_{6}\right)$. Here, $e_{1}, \ldots, e_{6}$ are the parameters of the fourth-rank tensor $\tilde{F}_{i j k l}\left(e_{1}, \ldots, e_{6}\right)$, which is the inverse of $\tilde{F}_{i j k l}\left(f_{1}, \ldots, f_{6}\right)$ with the following parameters

$$
\begin{aligned}
& f_{1}=S_{1}, f_{2}=S_{2}, f_{3}=S_{3}, \\
& f_{4}=S_{4}, f_{5}=\frac{1}{3}\left(\frac{\kappa_{0}}{\kappa_{2}-\kappa_{0}}-\frac{\mu_{0}}{\mu_{2}-\mu_{0}}\right)+S_{5}, f_{6}=\frac{1}{2} \frac{\mu_{0}}{\mu_{2}-\mu_{0}}+S_{6}
\end{aligned}
$$


After orientational averaging process, the averaged internal stresses of fibers $\subset \overline{\boldsymbol{\sigma}}_{1} \supset$ for randomly oriented fiber-reinforced composites can be obtained as

$$
\begin{aligned}
\subset \bar{\sigma}_{1} \supset & =\subset \mathbf{U} \supset: \overline{\boldsymbol{\epsilon}} \\
& =\subset U_{1} \supset \delta_{i j} \delta_{k l}+\subset U_{2} \supset\left(\delta_{i k} \delta_{j l}+\delta_{i l} \delta_{j k}\right)
\end{aligned}
$$

where

$$
\begin{aligned}
& \subset U_{1} \supset=\frac{1}{15}\left(U_{1}+5 U_{3}+5 U_{4}+15 U_{5}\right) \\
& \subset U_{2} \supset=\frac{1}{15}\left(U_{1}+10 U_{2}+15 U_{6}\right)
\end{aligned}
$$

In the case of tensile loading, the averaged internal stresses of fibers can be obtained as follows:

$$
\begin{aligned}
& \left(\bar{\sigma}_{11}\right)_{1}=\left[\subset U_{1} \supset+2 \subset U_{2} \supset\right] \bar{\epsilon}_{11}+\subset U_{1} \supset \bar{\epsilon}_{22}+\subset U_{1} \supset \bar{\epsilon}_{33} \\
& \left(\bar{\sigma}_{22}\right)_{1}=\subset U_{1} \supset \bar{\epsilon}_{11}+\left[\subset U_{1} \supset+2 \subset U_{2} \supset\right] \bar{\epsilon}_{22}+\subset U_{1} \supset \bar{\epsilon}_{33} \\
& \left(\bar{\sigma}_{33}\right)_{1}=\subset U_{1} \supset \bar{\epsilon}_{11}+\subset U_{2} \supset \bar{\epsilon}_{22}+\left[\subset U_{1} \supset+2 \subset U_{2} \supset\right] \bar{\epsilon}_{33}
\end{aligned}
$$

where $\bar{\epsilon}_{11}, \bar{\epsilon}_{22}$ and $\bar{\epsilon}_{33}$ are the total (ensemble-volume averaged) strains in the 1,2 and 3 directions, respectively. 


\section{FINITE ELEMENT IMPLEMENTATION}

\subsection{COMPUTATIONAL INTEGRATION ALGORITHMS}

Most existing literature pertaining to computational plasticity addresses one-phase ductile materials without inhomogeneities. On the other hand, most existing works in the micromechanics of composites are limited to monotonic and proportional loadings with simple power-law type, plastic strain-stress relationships. In this section, we employ the well-known strain-driven algorithm in which the stress history $\overline{\boldsymbol{\sigma}}$ is to be uniquely determined by the given strain history $\overline{\boldsymbol{\epsilon}}$. Similar to Ju and Tseng (1997), we will determine the unknown state of a local point $\left(\overline{\boldsymbol{\sigma}}_{n+1}, \overline{\boldsymbol{\epsilon}}_{n+1}^{p}, \bar{e}_{n+1}^{p}\right)$ at the end of the time step $t=t_{n+1}$ given the known state from the previous time step

$$
\left\{\overline{\boldsymbol{\epsilon}}_{n}, \overline{\boldsymbol{\epsilon}}_{n}^{p}, \bar{e}_{n}^{p}\right\} \quad \text { at } t=t_{n}
$$

where $\overline{\boldsymbol{\epsilon}}_{n}=$ the total (ensemble-volume averaged) strain; $\overline{\boldsymbol{\epsilon}}_{n}^{p}=$ the overall plastic strain; and $\bar{e}_{n}^{p}=$ the equivalent plastic strain, respectively, at time $t=t_{n}$. Based on the notion that the plastic strains are stress-free, the total stress $\overline{\boldsymbol{\sigma}}_{n}$ at time $t_{n}$ can be evaluated as

$$
\overline{\boldsymbol{\sigma}}_{n}=\mathbf{C}_{*}: \overline{\boldsymbol{\epsilon}}_{n}^{e}=\mathbf{C}_{*}:\left[\overline{\boldsymbol{\epsilon}}_{n}-\overline{\boldsymbol{\epsilon}}_{n}^{p}\right]
$$

Moreover, the current increment of the total strain $\Delta \overline{\boldsymbol{\epsilon}}_{n+1}$ is either given or can be obtained from the given strain-displacement history. Therefore, we have

$$
\overline{\boldsymbol{\epsilon}}_{n+1}=\overline{\boldsymbol{\epsilon}}_{n}+\Delta \overline{\boldsymbol{\epsilon}}_{n+1} ; \quad t_{n+1}=t_{n}+\Delta t_{n+1}
$$

where $\Delta t_{n+1}$ is the current time increment.

The two-step operator splitting methodology is adopted here to split the elastoplastic loading process into the elastic predictor and the plastic corrector. Given the solutions for the previous time step, an elastic predictor $\overline{\boldsymbol{\sigma}}_{n+1}^{t r}$ is calculated as

$$
\overline{\boldsymbol{\sigma}}_{n+1}^{t r}=\overline{\boldsymbol{\sigma}}_{n}+\mathbf{C}_{*}: \Delta \overline{\boldsymbol{\epsilon}}_{n+1}
$$

The evaluation of the yield function with the trial elastic stress and the previous hardening parameter $\left(\bar{\epsilon}_{n}^{p}\right)$ shows the status of the elastic predictor. Therefore, we write

$$
\subset \bar{F}_{n+1}^{t r}\left(\overline{\boldsymbol{\sigma}}_{n+1}^{t r}, \bar{e}_{n}^{p}\right) \supset=\left(1-\phi_{1}\right)^{2} \overline{\boldsymbol{\sigma}}_{n+1}^{t r}: \subset \overline{\mathbf{T}} \supset: \overline{\boldsymbol{\sigma}}_{n+1}^{t r}-K^{2}\left(\bar{e}_{n}^{p}\right)
$$

If $\subset \bar{F}_{n+1}^{t r} \supset \leq 0$, then the trial elastic stress remains either inside or on the yield surface. The incremental response is thus elastic, and no further plastic flow occurs for this strain increment. Consequently, we set $\overline{\boldsymbol{\sigma}}_{n+1}=\overline{\boldsymbol{\sigma}}_{n+1}^{t r}$ and $\bar{e}_{n+1}^{p}=\bar{e}_{n}^{p}$. 
In the case that $\subset \bar{F}_{n+1}^{t r} \supset>0$, the elastic predictor falls outside the yield surface, which is not allowed. Accordingly, the Kuhn-Tucker loading/unloading conditions must be enforced to bring the elastic predictor back to the yield surface. Here, we employ the implicit backward Euler method which is unconditionally stable. According to this method, the following approximations are made for the increments of the macroscopic plastic strain tensor $\overline{\boldsymbol{\epsilon}}_{n+1}^{p}$ and the equivalent plastic strain $\bar{e}_{n+1}^{p}$ :

$$
\begin{gathered}
\dot{\overline{\boldsymbol{\epsilon}}}_{n+1}^{p}=\frac{\overline{\boldsymbol{\epsilon}}_{n+1}^{p}-\overline{\boldsymbol{\epsilon}}_{n}^{p}}{\Delta t_{n+1}} \\
\dot{\bar{e}}_{n+1}^{p}=\frac{\bar{e}_{n+1}^{p}-\bar{e}_{n}^{p}}{\Delta t_{n+1}}
\end{gathered}
$$

By using Equations 172-173 and rearranging the terms in Equations 232-233, the updated values become:

$$
\begin{gathered}
\overline{\boldsymbol{\epsilon}}_{n+1}^{p}=\overline{\boldsymbol{\epsilon}}_{n}^{p}+\hat{\xi}_{n+1} \subset \overline{\mathbf{T}} \supset: \overline{\boldsymbol{\sigma}}_{n+1} \\
\bar{e}_{n+1}^{p}=\bar{e}_{n}^{p}+\hat{\xi}_{n+1} \sqrt{\frac{2}{3} \overline{\boldsymbol{\sigma}}_{n+1}: \subset \overline{\mathbf{T}} \supset: \overline{\boldsymbol{\sigma}}_{n+1}}
\end{gathered}
$$

with the following definition

$$
\hat{\xi}_{n+1} \equiv 2\left(1-\phi_{1}\right)^{2} \hat{\lambda}_{n+1} \equiv 2\left(1-\phi_{1}\right)^{2} \dot{\lambda} \Delta t_{n+1}
$$

As a result, the overall stress $\overline{\boldsymbol{\sigma}}_{n+1}$ can be calculated by using the elastic strain $\overline{\boldsymbol{\epsilon}}_{n+1}^{e}$ :

$$
\overline{\boldsymbol{\sigma}}_{n+1}=\mathbf{C}_{*}: \overline{\boldsymbol{\epsilon}}_{n+1}^{e}=\mathbf{C}_{*}:\left[\overline{\boldsymbol{\epsilon}}_{n+1}-\overline{\boldsymbol{\epsilon}}_{n+1}^{p}\right]
$$

By substituting Equation 235 into 237, we arrive at

$$
\overline{\boldsymbol{\sigma}}_{n+1}=\mathbf{C}_{*}:\left[\overline{\boldsymbol{\epsilon}}_{n+1}-\overline{\boldsymbol{\epsilon}}_{n}^{p}-\hat{\xi}_{n+1} \subset \overline{\mathbf{T}} \supset: \overline{\boldsymbol{\sigma}}_{n+1}\right]
$$

From the Kuhn-Tucker conditions, the yield function given in Equation 121 must be always zero during the plastic loading process. Therefore, the parameter $\hat{\xi}_{n+1}$ can be evaluated by enforcing the plastic consistency condition at $t=t_{n+1}$ :

$$
\subset \bar{F}_{n+1}\left(\hat{\xi}_{n+1}\right) \supset=\left(1-\phi_{1}\right)^{2} \overline{\boldsymbol{\sigma}}_{n+1}: \subset \overline{\mathbf{T}} \supset: \overline{\boldsymbol{\sigma}}_{n+1}-K^{2}\left(\bar{e}_{n+1}^{p}\right)=0
$$

Substitution of Equations 237 and 238 into Equation 239 renders a nonlinear scalar equation in terms of $\hat{\xi}_{n+1}$. Once the parameter $\hat{\xi}_{n+1}$ is numerically solved from Equation 239, the total current stress, the plastic strain, and the hardening parameter are updated according to Equation 238, 234, and 235. 
For convenience, Exhibits 1 and 2 summarize the above micromechanical iterative computational algorithm for the elastoplastic behavior of three-phase RFPCs accounting for interfacial damage evolution. In particular, Exhibit 1 renders a step-by-step flow chart for the computational procedure to determine the current perfectly bonded fibers volume fraction $\left(\phi_{1}\right)_{n+1}$ in accordance with the evolutionary interfacial debonding model presented in Section 3.3. Furthermore, Exhibit 2 provides a detail iterative return mapping algorithm consistent with this section.

\subsection{CONTINUUM TANGENT MODULI}

In solving the nonlinear programs by the finite element method, the matrix of global tangent moduli needs to be constructed for the global Newton's iteration. The Newton's iteration should have a quadratic rate of convergence if proper tangent is used during the iteration. The continuum tangent moduli is derived from the rate equations without considering the numerical discretization traditionally used in finite element analysis programs. In this section, the continuum tangent moduli for the rate-independent plasticity for the RFPCsCFPMCs is derived based on the rate equations for the proposed micromechanics based formulation.

For convenience, let us rephrase Equations 172 and 173 as follows:

$$
\begin{aligned}
& \dot{\overline{\boldsymbol{\epsilon}}}^{p}=\dot{\lambda} \overline{\mathbf{r}} \\
& \dot{\bar{e}}^{p}=\dot{\lambda} \overline{\mathbf{h}}
\end{aligned}
$$

where the following definitions have been made

$$
\begin{gathered}
\overline{\mathbf{r}} \equiv \frac{\partial \subset \bar{F} \supset}{\partial \overline{\boldsymbol{\sigma}}}=2\left(1-\phi_{1}\right)^{2} \subset \overline{\mathbf{T}} \supset: \overline{\boldsymbol{\sigma}} \\
\overline{\mathbf{h}} \equiv \sqrt{\frac{2}{3} \frac{\partial \subset \bar{F} \supset}{\partial \overline{\boldsymbol{\sigma}}}: \subset \overline{\mathbf{T}} \supset^{-1}: \frac{\partial \subset \bar{F} \supset}{\partial \overline{\boldsymbol{\sigma}}}}=2\left(1-\phi_{1}\right)^{2} \sqrt{\frac{2}{3} \overline{\boldsymbol{\sigma}}: \subset \overline{\mathbf{T}} \supset: \overline{\boldsymbol{\sigma}}}
\end{gathered}
$$

By taking the time derivative on Equation 74 and combining Equations 73 and 240, we have

$$
\dot{\overline{\boldsymbol{\sigma}}}=\mathbf{C}_{*}:\left[\dot{\overline{\boldsymbol{\epsilon}}}-\dot{\overline{\boldsymbol{\epsilon}}}^{p}\right]=\mathbf{C}_{*}:[\dot{\overline{\boldsymbol{\epsilon}}}-\dot{\lambda} \overline{\mathbf{r}}]
$$

where the parameter $\dot{\lambda}$ is solved from the consistency condition $\subset \dot{\bar{F}} \supset=0$. According to the chain rule, the plasticity condition leads to

$$
\subset \dot{\bar{F}}\left(\overline{\boldsymbol{\sigma}}, \bar{e}^{p}\right) \supset=\frac{\partial \subset \bar{F} \supset}{\partial \overline{\boldsymbol{\sigma}}}: \dot{\overline{\boldsymbol{\sigma}}}+\frac{\partial \subset \bar{F} \supset}{\partial \bar{e}^{p}} \dot{\overline{e^{p}}}=0
$$

which yields the following equation upon the substitution of Equations 241 and 244 into 245

$$
\frac{\partial \subset \bar{F} \supseteq}{\partial \overline{\boldsymbol{\sigma}}}: \mathbf{C}_{*}:[\dot{\overline{\boldsymbol{\epsilon}}}-\dot{\lambda} \bar{r}]+\dot{\lambda} \frac{\partial \subset \bar{F} \supseteq}{\partial \bar{e}^{p}} \bar{h}=0
$$


Exhibit 1. Micromechanical iterative algorithm for the evolutionary damage model.

(i) Estimate model parameters :

Plastic material parameters : $\sigma_{y}, h, \bar{q} ;$ Weibull parameters : $S_{o}, M$

(ii) Initialize : set $z=0 ;\left(\phi_{1}\right)_{n+1}^{(0)}=\left(\phi_{1}\right)_{n},\left(\phi_{2}\right)_{n+1}^{(0)}=\left(\phi_{2}\right)_{n}$

(iii) Compute :

Coefficients : $\subset \overline{\mathbf{T}}_{n+1}^{(z)} \supset, \mathbf{U}_{n+1}^{(z)}$; Effective moduli : $\left(\kappa_{*}\right)_{n+1}^{(z)},\left(\mu_{*}\right)_{n+1}^{(z)},\left(E_{*}\right)_{n+1}^{(z)},\left(\nu_{*}\right)_{n+1}^{(z)}$

(iv) Compute internal stresses of fibers :

$$
\begin{aligned}
& {\left[\left(\bar{\sigma}_{11}\right)_{1}\right]_{n+1}^{(z)}=\left[\left(U_{1}\right)_{n+1}^{(z)}+2\left(U_{2}\right)_{n+1}^{(z)}\right]\left(\bar{\epsilon}_{11}\right)_{n+1}+\left(U_{1}\right)_{n+1}^{(z)}\left(\bar{\epsilon}_{22}\right)_{n+1}+\left(U_{1}\right)_{n+1}^{(z)}\left(\bar{\epsilon}_{33}\right)_{n+1}} \\
& {\left[\left(\bar{\sigma}_{22}\right)_{1}\right]_{n+1}^{(z)}=\left(U_{1}\right)_{n+1}^{(z)}\left(\bar{\epsilon}_{11}\right)_{n+1}+\left[\left(U_{1}\right)_{n+1}^{(z)}+2\left(U_{2}\right)_{n+1}^{(z)}\right]\left(\bar{\epsilon}_{22}\right)_{n+1}+\left(U_{1}\right)_{n+1}^{(z)}\left(\bar{\epsilon}_{33}\right)_{n+1}} \\
& {\left[\left(\bar{\sigma}_{33}\right)_{1}\right]_{n+1}^{(z)}=\left(U_{1}\right)_{n+1}^{(z)}\left(\bar{\epsilon}_{11}\right)_{n+1}+\left(U_{1}\right)_{n+1}^{(z)}\left(\bar{\epsilon}_{22}\right)_{n+1}+\left[\left(U_{1}\right)_{n+1}^{(z)}+2\left(U_{2}\right)_{n+1}^{(z)}\right]\left(\bar{\epsilon}_{33}\right)_{n+1}}
\end{aligned}
$$

(v) Compute the Weibull probability distribution function :

$$
P_{d}\left\{\left[\left(\bar{\sigma}_{11}\right)_{1}\right]_{n+1}^{(z)}\right\}=1-\exp \left[-\left(\frac{\left[\left(\bar{\sigma}_{11}\right)_{1}\right]_{n+1}^{(z)}}{S_{o}}\right)^{M}\right]
$$

(vi) Compute volume fractions :

$$
\begin{gathered}
\left(\phi_{2}\right)_{n+1}^{(z)}=\phi P_{d}\left\{\left[\left(\bar{\sigma}_{11}\right)_{1}\right]_{n+1}^{(z)}\right\}=\phi\left\{1-\exp \left[-\left(\frac{\left[\left(\bar{\sigma}_{11}\right)_{1}\right]_{n+1}^{(z)}}{S_{o}}\right)^{M}\right]\right\}, \\
\left(\phi_{1}\right)_{n+1}^{(z)}=\phi-\left(\phi_{2}\right)_{n+1}^{(z)}
\end{gathered}
$$

(vii) Perform convergence check :

If $\left|\frac{\left(\phi_{1}\right)_{n+1}^{(z)}-\left(\phi_{1}\right)_{n+1}^{(z-1)}}{\left(\phi_{1}\right)_{n+1}^{(z-1)}}\right| \leq T O L \quad\left(e . g ., 10^{-8}\right):$ then update quantities in (iii), (iv), (vi)

$$
\begin{aligned}
& \subset \overline{\mathbf{T}}_{n+1} \supset=\subset \overline{\mathbf{T}}_{n+1}^{(z)} \supset, \ldots,\left(\nu_{*}\right)_{n+1}=\left(\nu_{*}\right)_{n+1}^{(z)} ;\left[\left(\bar{\sigma}_{i i}\right)_{1}\right]_{n+1}=\left[\left(\bar{\sigma}_{i i}\right)_{1}\right]_{n+1}^{(z)}, \quad(i=1,2,3) ; \\
& \left(\phi_{1}\right)_{n+1}=\left(\phi_{1}\right)_{n+1}^{(z)},\left(\phi_{2}\right)_{n+1}=\left(\phi_{2}\right)_{n+1}^{(z)} ; \quad \text { GO TO (viii) in Exhibit 2. }
\end{aligned}
$$

Otherwise : $\operatorname{SET} z=z+1$; GO TO (iii). 
Exhibit 2. 3-D return mapping algorithm.

(viii) 3D return mapping algorithm :

(a) Initialize : set $l=0 ;\left(\overline{\boldsymbol{\epsilon}}_{n+1}^{p}\right)^{(0)}=\overline{\boldsymbol{\epsilon}}_{n}^{p},\left(\bar{e}_{n+1}^{p}\right)^{(0)}=\bar{e}_{n}^{p}$ (for local Newton iteration)

(b) Compute : $\overline{\boldsymbol{\sigma}}_{n}=\mathbf{C}_{n}:\left[\overline{\boldsymbol{\epsilon}}_{n}-\overline{\boldsymbol{\epsilon}}_{n}^{p}\right]$

(c) Compute the elastic predictor :

Trial elastic state $: \overline{\boldsymbol{\sigma}}_{n+1}^{t r}=\overline{\boldsymbol{\sigma}}_{n}+\mathbf{C}_{n}: \Delta \boldsymbol{\epsilon}_{n+1}$

Compute : $\overline{\boldsymbol{\epsilon}}_{n+1}=\overline{\boldsymbol{\epsilon}}_{n}+\Delta \boldsymbol{\epsilon}_{n+1}$;

$$
\subset \bar{F}_{n+1}^{t r}\left(\overline{\boldsymbol{\sigma}}_{n+1}^{t r}, \bar{e}_{n}^{p}\right) \supset=\left(1-\left(\phi_{1}\right)_{n+1}\right)^{2} \overline{\boldsymbol{\sigma}}_{n+1}^{t r}: \subset \overline{\mathbf{T}}_{n+1} \supset: \overline{\boldsymbol{\sigma}}_{n+1}^{t r}-K^{2}\left(\bar{e}_{n}^{p}\right)
$$

(d) Check whether plastic loading is active :

If $\subset \bar{F}_{n+1}^{t r} \supset \leq T O L$ (elastic step; e.g., $T O L=10^{-8}$ ) :

$$
\hat{\xi}_{n+1}=0 ; \text { SET } \overline{\boldsymbol{\sigma}}_{n+1}=\overline{\boldsymbol{\sigma}}_{n+1}^{t r}, \bar{e}_{n+1}^{p}=\bar{e}_{n}^{p} ; \text { GO TO (iii) in Exhibit } 1 .
$$

Otherwise (plastic step) : GO TO (e).

(e) Perform plastic correction; return mapping algorithm :

Solve nonlinear scalar equation for $\hat{\xi}_{n+1}$ : use local Newton iteration

$$
\subset \bar{F}_{n+1}\left(\hat{\xi}_{n+1}^{(l)}\right) \supset=\left[1-\left(\phi_{1}\right)_{n+1}\right]^{2} \overline{\boldsymbol{\sigma}}_{n+1}^{(l)}: \subset \overline{\mathbf{T}}_{n+1} \supset: \overline{\boldsymbol{\sigma}}_{n+1}^{(l)}-K^{2}\left[\left(\bar{e}_{n+1}^{p}\right)^{(l)}\right]=0
$$

(f) Perform convergence check:

If $\left|\subset \bar{F}_{n+1}\left[\left(\hat{\xi}_{n+1}\right)^{(l)}\right] \supset\right| \leq T O L\left(e . g ., 10^{-8}\right):$ then update

$$
\hat{\xi}_{n+1}=\hat{\xi}_{n+1}^{(l)}, \overline{\boldsymbol{\sigma}}_{n+1}=\overline{\boldsymbol{\sigma}}_{n+1}^{(l)}, \overline{\boldsymbol{\epsilon}}_{n+1}^{p}=\left(\overline{\boldsymbol{\epsilon}}_{n+1}^{p}\right)^{(l)}, \bar{e}_{n+1}^{p}=\left(\bar{e}_{n+1}^{p}\right)^{(l)} ; \text { EXIT. }
$$

Otherwise : SET $l=l+1$

Compute derivative of $\subset \bar{F}\left[\left(\hat{\xi}_{n+1}\right)^{(l)}\right] \supset: D \subset \bar{F}\left[\left(\hat{\xi}_{n+1}\right)^{(l)}\right] \supset$

$$
\left(\hat{\xi}_{n+1}\right)^{(l+1)}=\left(\hat{\xi}_{n+1}\right)^{(l)}-\frac{\subset \bar{F}\left[\left(\hat{\xi}_{n+1}\right)^{(l)}\right] \supset}{D \subset \bar{F}\left[\left(\hat{\xi}_{n+1}\right)^{(l)}\right] \supset} ; \quad \text { GO TO (e). }
$$


Therefore, the parameter $\dot{\lambda}$ is obtained from the above equation and is written as

$$
\dot{\lambda}=\left[\frac{\partial \subset \bar{F} \supset}{\partial \overline{\boldsymbol{\sigma}}}: \mathbf{C}_{*}: \bar{r}-\frac{\partial \subset \bar{F} \supset}{\partial \bar{e}^{p}}\right]^{-1} \frac{\partial \subset \bar{F} \supset}{\partial \overline{\boldsymbol{\sigma}}}: \mathbf{C}_{*}: \dot{\bar{\epsilon}}
$$

Furthermore, by substituting Equation 247 into Equation 244, the following equation which defines the continuum tangent moduli is obtained:

$$
\dot{\overline{\boldsymbol{\sigma}}}=\mathbf{C}_{\text {const }}^{e p}: \dot{\overline{\boldsymbol{\epsilon}}}=\left\{\mathbf{C}_{*}-\left[\frac{\partial \subset \bar{F} \supset}{\partial \overline{\boldsymbol{\sigma}}}: \mathbf{C}_{*}: \overline{\mathbf{r}}-\frac{\partial \subset \bar{F} \supset}{\partial \bar{e}^{p}} \bar{h}\right]^{-1}\left(\mathbf{C}_{*}: \overline{\mathbf{r}}\right) \otimes\left(\mathbf{C}_{*}: \frac{\partial \subset \bar{F} \supset}{\partial \overline{\boldsymbol{\sigma}}}\right)\right\}: \dot{\overline{\boldsymbol{\epsilon}}}
$$

Moreover, the explicit form of the symmetrical fourth-rank tensor $\mathbf{C}_{c o n s t}^{e p}$ can be obtained by substituting the definitions of $\overline{\mathbf{r}}$ and $\bar{h}$ in Equations 242 and 243 into Equation 248:

$$
\mathbf{C}_{\text {const }}^{e p} \equiv \frac{\left(\mathbf{C}_{*}: \subset \overline{\mathbf{T}} \supset: \overline{\boldsymbol{\sigma}}\right) \otimes\left(\mathbf{C}_{*}: \subset \overline{\mathbf{T}} \supset: \overline{\boldsymbol{\sigma}}\right)}{(\overline{\boldsymbol{\sigma}}: \subset \overline{\mathbf{T}} \supset): \mathbf{C}_{*}:(\subset \overline{\mathbf{T}} \supset: \overline{\boldsymbol{\sigma}})-\left(1-\phi_{1}\right)^{-1} \sqrt{\frac{2}{3}} K^{\prime} \overline{\boldsymbol{\sigma}}: \subset \overline{\mathbf{T}} \supset: \overline{\boldsymbol{\sigma}}}
$$

in which $K^{\prime} \equiv \frac{\partial K\left(e^{\bar{p}}\right)}{\partial e^{\bar{p}}}$. It is noted that the requirement of $\subset \bar{F} \supset=0$ has been used in deriving Equation 249 . 


\section{NUMERICAL SIMULATIONS AND EXPERIMENTAL COMPARISON}

The numerical and experimental studies to characterize damage evolution in discontinuous, random fiber-reinforced composites have been limited in the literature until now. One such experimental and numerical study on discontinuous, random fiber-reinforced composites was made by Meraghni and Benzeggagh (1995) and Meraghni et al. (1996). Their modeling relied on an experimental approach, developed through a methodology of experimental identification of basic damage mechanisms, which involves amplitude analysis of acoustic emission and microscopic observations. They introduced an experimental damage parameter $\beta$ to allow the modeling of damage mechanisms. A micromechanics-based analytical simulation was also presented and compared with the experimental data.

To illustrate the elastoplastic behavior of the present damage constitutive framework, our present damage model considering interfacial debonding is exercised in Figures 3 - 5. The material properties of RFPCs involving these simulations are $E_{0}=3.0 G P a, \nu_{0}=0.35, E_{1}=380 G P a, \nu_{1}=0.25, \sigma_{y}=125 M P a, h=$ $400 M P a$ and $\bar{q}=0.5$. In addition, to implement the proposed probabilistic micromechanics based on Weibull function into the present constitutive models, we need to estimate the values of Weibull partameters $S_{o}$ and $M$. For simplicity, we assume the Weibull parameters to be $S_{o}=75 * \sigma_{s}^{p}$ and $M=40$. Figure 3 shows the effect of the shape of fibers on the mechanical behavior of RFPCs with the same volume fraction of fibers. It is clear that the elastoplastic behavior of the composites is strongly dependent upon the shape of fibers, especially for the plastic stage. Figures 4 and 5 exhibit the effect of the initial volume fraction of fibers on the behavior and progressive debonding of the composites. It is seen that the composite with high initial volume fraction of fibers is stiffer, but the influence of damage on the stress-strain response of the composite is more drastic because of quick damage evolution.

To further assess the present damage constitutive models, we now compare the present predictions with experimental data reported by Mergahni and Benzeggagh (1995) for uniaxial stress-strain behavior of randomly oriented, discontinuous glass fiber epoxy composites. Here, we adopt the elastic properties, aspect ratio and fiber volume fraction according to Mergahni and Benzeggagh (1995) as follows: $E_{0}=3.0 G P a, \nu_{0}=$ $0.35, E_{1}=72 G P a, \nu_{1}=0.17, \alpha=19.25$ and $\phi_{1}=0.5$. Followed by the parameter estimation algorithm developed by Ju et al. (1987) and Simo et al. (1988), we estimate the plastic parameters $\sigma_{y}, h$ and $\bar{q}$ in accordance with the isotropic hardening law given in Equation 176 and Weibull parameters $S_{o}$ and $M$ for evolutionary debonding to be $\sigma_{y}=150 M P a, h=400 M P a, \bar{q}=0.5 ; S_{o}=27.14 * \sigma_{y}$ and $M=4.0$. We depict our prediction against the experimental data provided by Mergahni and Benzeggagh (1995) in Figures 6 and 7. Since our formulation does not consider inter-fiber interaction, the stress-strain curve for the present 
prediction is lower than that based on the experiment in the early stage. Naturally, the overall stiffness of interacting damage model is higher than that of noninteracting damage model (Lee, 1998). As the strain increases, the effect of damage becomes dominant one, and the curves corresponding the present prediction and the experiment will intersect each other, because the proposed damage constitutive model includes the interfacial debonding only. Therefore, it is concluded that the interaction effect among constituents must be considered in modeling damage behavior of composites for both moderately and extremely high fiber volume fraction. Furthermore, other damage mechanisms (e.g. matrix cracking, void nucleation, etc.) must be included in our damage constitutive models to offer more realistic damage predictions. Finally, the present model does not account for other damage mechanisms nor impact, since these effects are beyond the scope of the present stage of this research. In spite of these limitations, the agreement between the present predictions and experiments is encouraging for possible use of the proposed damage constitutive models for predicting the progressive damage in composite structures. 


\section{FUTURE RESEARCH}

Future research will be needed to extend the proposed damage constitutive model to practical crushing problems. In addition, further assessment and experimental validation of the proposed framework are also needed in the future.

When the spheroidal (prolate) fibers are randomly oriented in the 1-2 plane, the composite as a whole is transversely isotropic. Such a system exists in sheet molding compounds (SMC), which is close to the manufacturing process of RFPCs, among others. The implementation of two-dimensional orientation averaging process into the present model is straightforward and will be included based on the orientation averaging process proposed in this study.

Composite structures may undergo compression, tension and/or combined failures, depending on the specimen configuration, under impact loading. Accordingly, a new failure criterion based on experimental verifications for randomly oriented, discontinuous fiber-reinforced composites will be developed to perform failure analysis of RFPCs. The effective moduli of microcrack-weakened polymeric composites will be also proposed based on Lee's (1998) approximate scheme to address the effect of microcracks on the overall stiffness and strength of the composites.

Moreover, when fiber volume fraction is higher, strong fiber (mechanical) interactions become significant. Excellent strong fiber interaction models were proposed by Ju and Tseng (1996) and Lee (1998) for pairwise inclusion interactions at the constitutive level. In accordance with their approximate solution of a micromechanical damage constitutive model considering the pairwise fiber interaction, the present damage constitutive model will be extended and will be able to account for the effect of interactions for the composite with high fiber volume fraction. It is emphasized that the approximate, close-form analytical damage constitutive model will provide a method for obtaining second-order, ensemble-volume averaged perturbations due to the existence and the interaction of fibers.

Finally, the complete progressive damage constitutive models, in conjunction with structural testing and microscope observations to identify and classify the complex damage mechanism, will be implemented into finite element code, DYNA3D, to solve the large scale problems, such as automobile components and systems. Furthermore, crush tests for the composite tube will be performed to determine the validity of the current damage constitutive models. 


\section{REFERENCES}

1. M. Caslini, C. Zanotti, and T. K. O'Brien. Study of matrix cracking and delamination in glass/epoxy laminates. Journal of Composites Technology and Research, 9:121-130, 1987.

2. H. Y. Choi and F. K. Chang. A model for predicting damage in graphite/epoxy laminated composites resulting from low-velocity point impact. Journal of Composite Materials, 26:2134-2169, 1992.

3. R. M. Christensen and S. R. Swanson. Evaluation of a new failure criterion for fibrous composite materials. In Mechanics of Composites Materials, AMD-Vol, 92:63-68, 1988.

4. J. Donnet and R. C. Bansal. Carbon fibers, Marcel Dekker, New York and Basel, 1990.

5. J. D. Eshelby. The determination of the elastic field of an ellipsoidal inclusion, and related problems. Proc. R. Soc. Lond., A241:376-396, 1957.

6. J. D. Eshelby. The elastic field outside an ellipsoidal inclusion. Proc. R. Soc. Lond., A252:561-569, 1959.

7. J. D. Eshelby. Elastic inclusions and inhomogeneities. In Progress in Solid Mechanics, II, 87-140, North-Holland, Amsterdam, 1961.

8. J. N. Goodier. On the integration of the thermo-elastic equations. Phil. Mag., 23:1017-1032, 1937.

9. S. E. Groves, C. E. Harris, A. L. Highsmith, D. H. Allen and R. G. Norvell. An experimental and analytical treatment of matrix cracking in cross-ply laminates. Experimental Mechanics, 27:73-79, 1987.

10. H. T. Hahn, J. B. Erikson, and S. W. Tsai. Characterization of matrix/interface controlled strength of unidirectional composites. In Fracture of composite materials, Martinus Nijhoff, 197-214, 1982.

11. Z. Hashin. Failure criteria for unidirectional fiber composites. J. Appl. Mech., 47:329-334, 1980.

12. R. Hill. Theory of mechanical properties of fiber-strengthened materials: I. Elastic behavior. J. Mech. Phys. Solids, 12:199-212, 1964.

13. J. W. Ju and T. M. Chen. Micromechanics and effective moduli of elastic composites containing randomly dispersed ellipsoidal inhomogeneities. Acta Mechanica, 103:103-121, 1994a.

14. J. W. Ju and T. M. Chen. Effective elastic moduli of two-phase composites containing randomly dispersed spherical inhomogeneities. Acta Mechanica, 103:123-144, 1994b.

15. J. W. Ju and H. K. Lee. A micromechanical damage model for effective elastoplastic behavior of ductile matrix composites considering evolutionary complete particle debonding. Journal of Computer Meth. in Appl. Mech. and Eng., in press, 1999. 
16. J.W. Ju, J. C. Simo, K. S. Pister, and R. L. Taylor. A parameter estimation algorithm for inelastic material models. In Proceedings of the Second Int. Conf. on Constitutive Laws for Engineering Materials: Theory and Application, 1233-1240, University of Arizona, 1987.

17. J. W. Ju and L. Z. Sun. A novel formulation for the exterior-point Eshelby's tensor of an ellipsoidal inclusion. J. Appl. Mech., 66:570-574, 1999.

18. J. W. Ju and K. H. Tseng. Effective elastoplastic behavior of two-phase ductile matrix composites: A micromechanical framework. Int. J. Solids Structures, 33:4267-4291, 1996.

19. J. W. Ju and K. H. Tseng. Effective elastoplastic algorithm for ductile matrix composites. J. Eng. Mech., 123:260-266, 1997.

20. R. Keal. Post failure energy absorbing mechanism of filament wound composite tubes. Ph.D. Dissertation, University of Liverpool, 1983.

21. Z. Kutlu and F. K. Chang. Composite panels containing multiple through-the-width delaminations and subjected to compression. Part I: analysis. Composite Structures, 31:273-296, 1995.

22. H. K. Lee. Three-dimensional micromechanical damage models for effective elastic and elastoplastic behavior of composite materials with inhomogeneities or microcracks. Ph.D. Dissertation, University of California, Los Angeles, 1998.

23. A. Matzenmiller and K. Schweizerhof. Crashworthiness simulations of composite structures - a first step with explicit time integration. CAD-FEM GmbH, Munchen, 1991.

24. F. Meraghni and M. L. Benzeggagh. Micromechanical modeling of matrix degradation in randomly discontinuous-fibre composites. Composites Science and Technology, 55:171-186, 1995.

25. F. Meraghni, C. J. Blakeman, and M. L. Benzeggagh. Effect of interfacial decohesion on stiffness reduction in a random discontinuous-fibre composite containing matrix microcracks. Composites Science and Technology, 56:541-555, 1996.

26. T. Mura. Micromechanics of defects in solids., Martinus Nijhoff, The Hague, The Netherlands, 1987.

27. T. Mura, H. M. Shodja, and Y. Hirose. Inclusion problems. Appl. Mech. Rev., 49:S118-127, 1996.

28. Y. D. Murray. Theory and verification of the fiber composite damage model implemented in DYNA3D. Technical Report, APTEC, San Jose, 1989.

29. S. Nemat-Nasser and M. Hori. Micromechanics: Overall Properties of Heterogeneous Materials. North-Holland, Amsterdam, 1993. 
30. J. N. Price and D. Hull. Axial crushing of glass fiber-polyester composite cones. Composites Science and Technology, 28:211-230, 1987.

31. R. E. Robertson and V. E. Mindroiu. Discontinuous fiber composites. Engineering Materials Handbook, Volume I: Composites, ASM International, 794-797, 1987.

32. S. Simunovic, S. and T. Zacharia. Application of high performance computing to automotive design and manufacturing: Composite materials modeling task technical manual for constitutive models for glass fiber-polymer matrix composites. Technical Report ORNL/TM-13444, Oak Ridge National Laboratory, 1997.

33. G. P. Tandon and G. J. Weng. Average stress in the matrix and effective moduli of randomly oriented composites. Composites Science and Technology, 27:111-132, 1986.

34. K. Tohgo and G. J. Weng. A progress damage mechanics in particle-reinforced metal-matrix composites under high triaxial tension. J. Eng. Mat. Tech., 116:414-420, 1994.

35. A. S. D. Wang. Fracture Mechanics of sublaminate cracks in composite materials. Composites Technology, 6:45-62, 1984.

36. W. Weibull. A statistical distribution function of wide applicability. J. of Appl. Mech., 18:293-297, 1951.

37. Y. H. Zhao Y. H. and G. J. Weng. A theory of inclusion debonding and its influence on the stress-strain relations of a ductile matrix. International Journal of Damage Mechanics, 4:196-211, 1995.

38. Y. H. Zhao and G. J. Weng. Plasticity of a two-phase composite with partially debonded inclusions. International Journal of Plasticity, 12:781-804, 1996.

39. Y. H. Zhao and G. J. Weng. Transversely isotropic moduli of two partially debonded composites. Int. J. Solids Structures, 34:493-507, 1997. 
APPENDIX A. FIGURES 


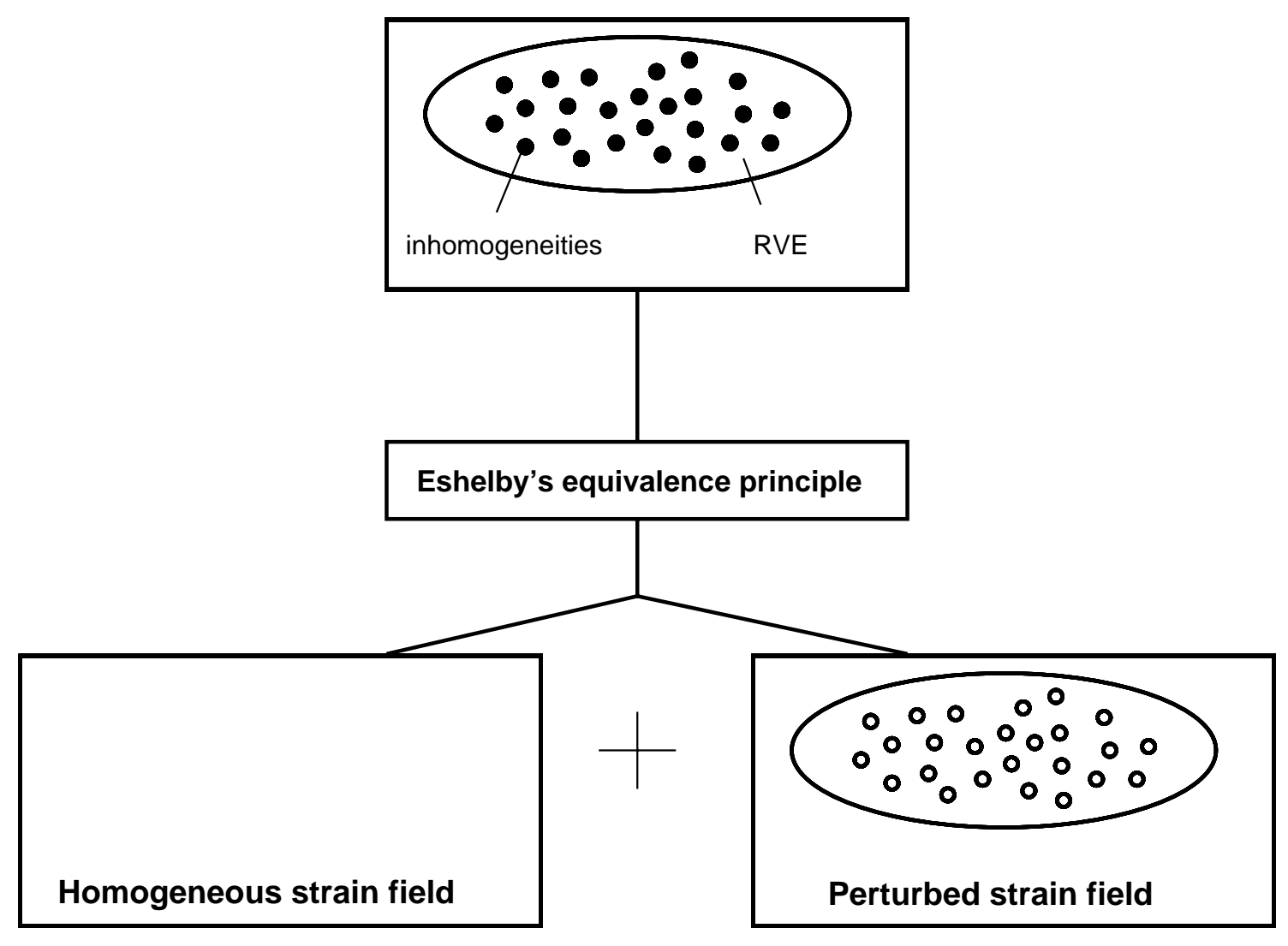

Figure 1. An RVE for a composite and superposition involving homogeneous stran and perturbed strain. 


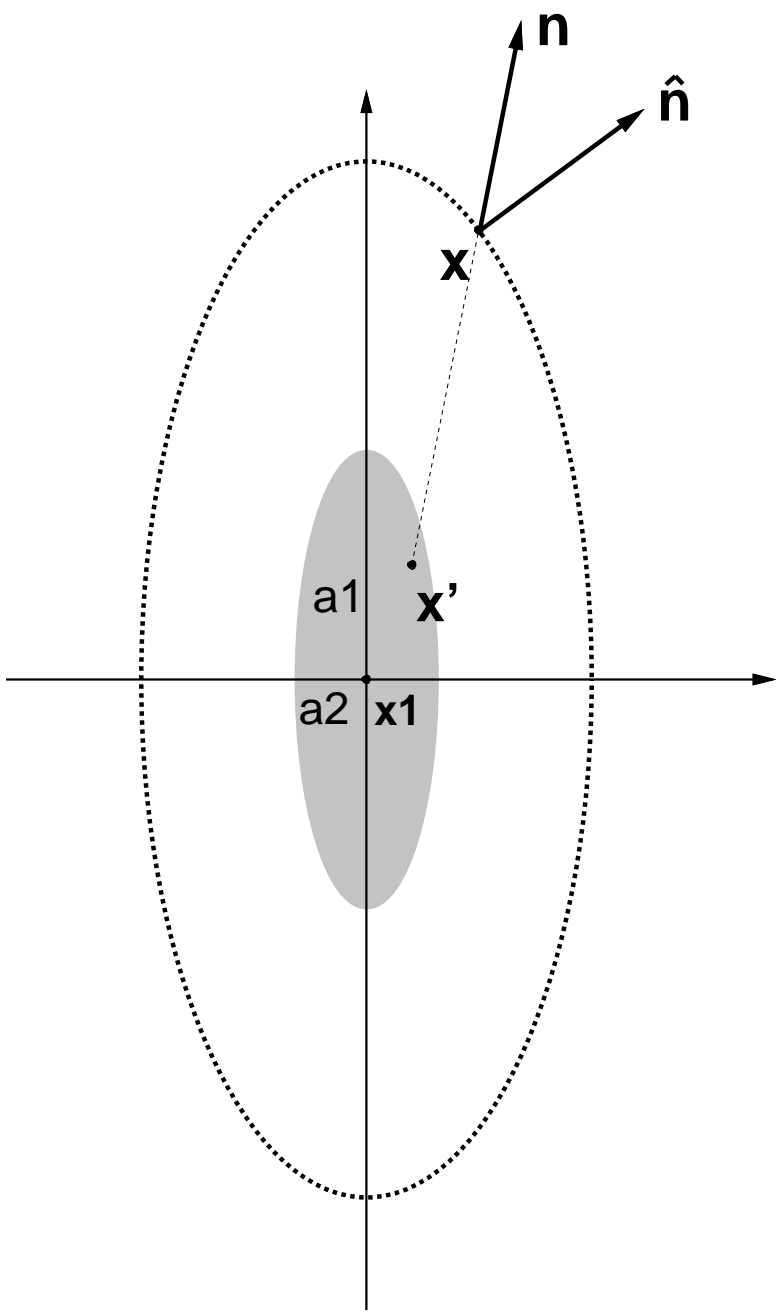

Figure 2. Schematic description of the imaginary ellipsoid and its outward unit normal vector for an ellipsoidal inclusion. 


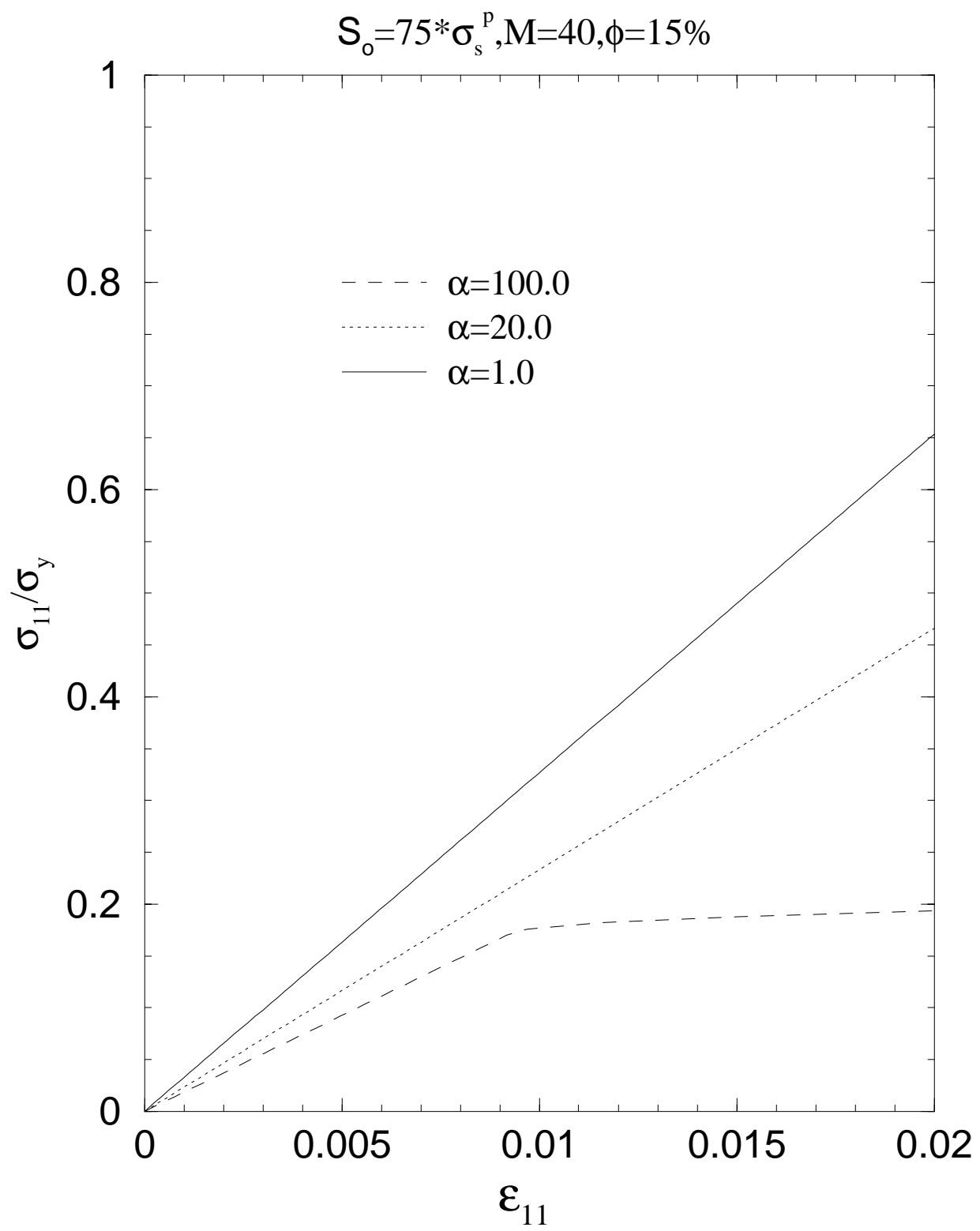

Figure 3. Effect of the shape of fibers on the overall uniaxial elastoplastic behavior of random carbon fiber polymer matrix composites. 


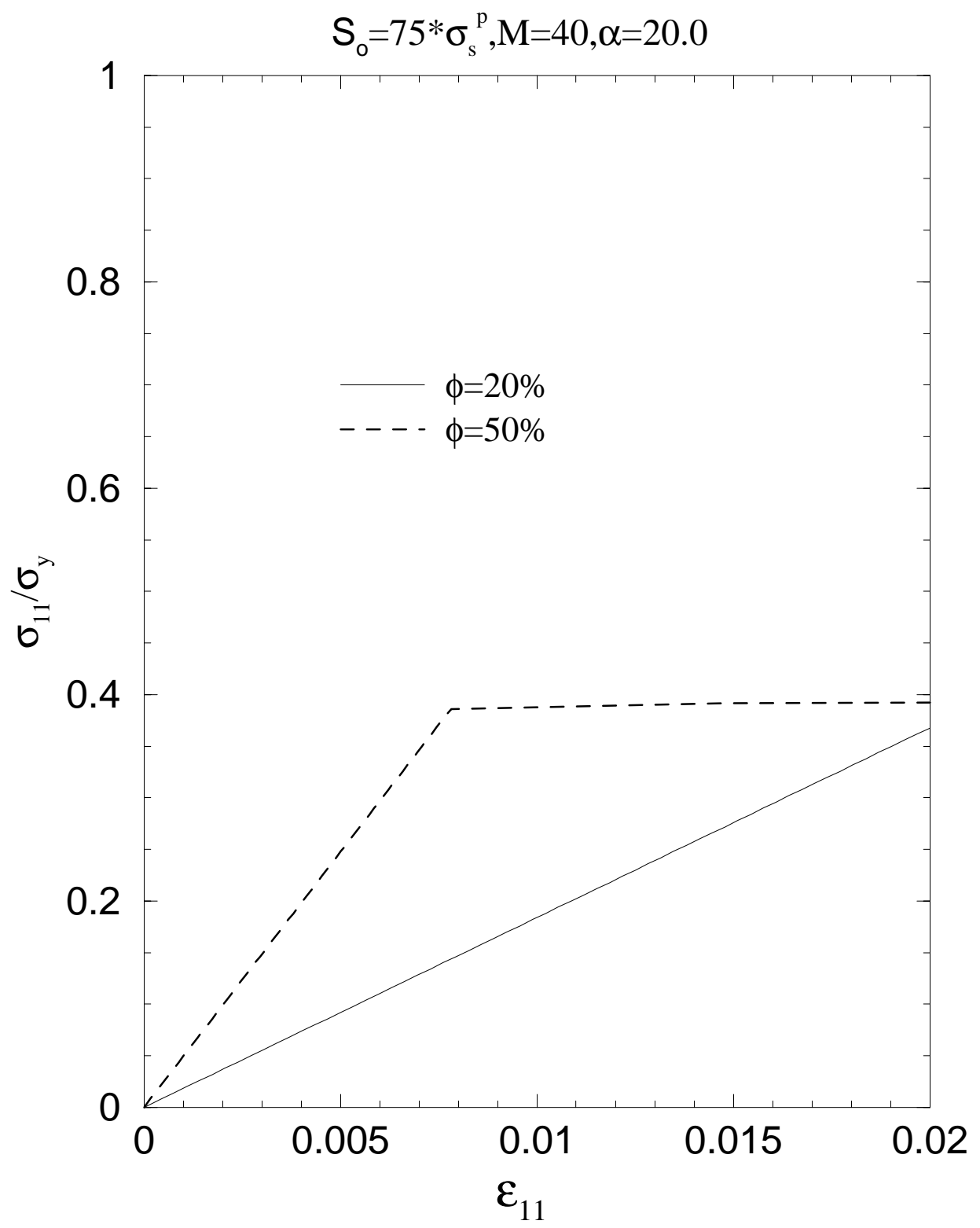

Figure 4. Effect of the initial volume fraction of fibers on the overall uniaxial elastoplastic behavior of random carbon fiber polymer matrix composites. 


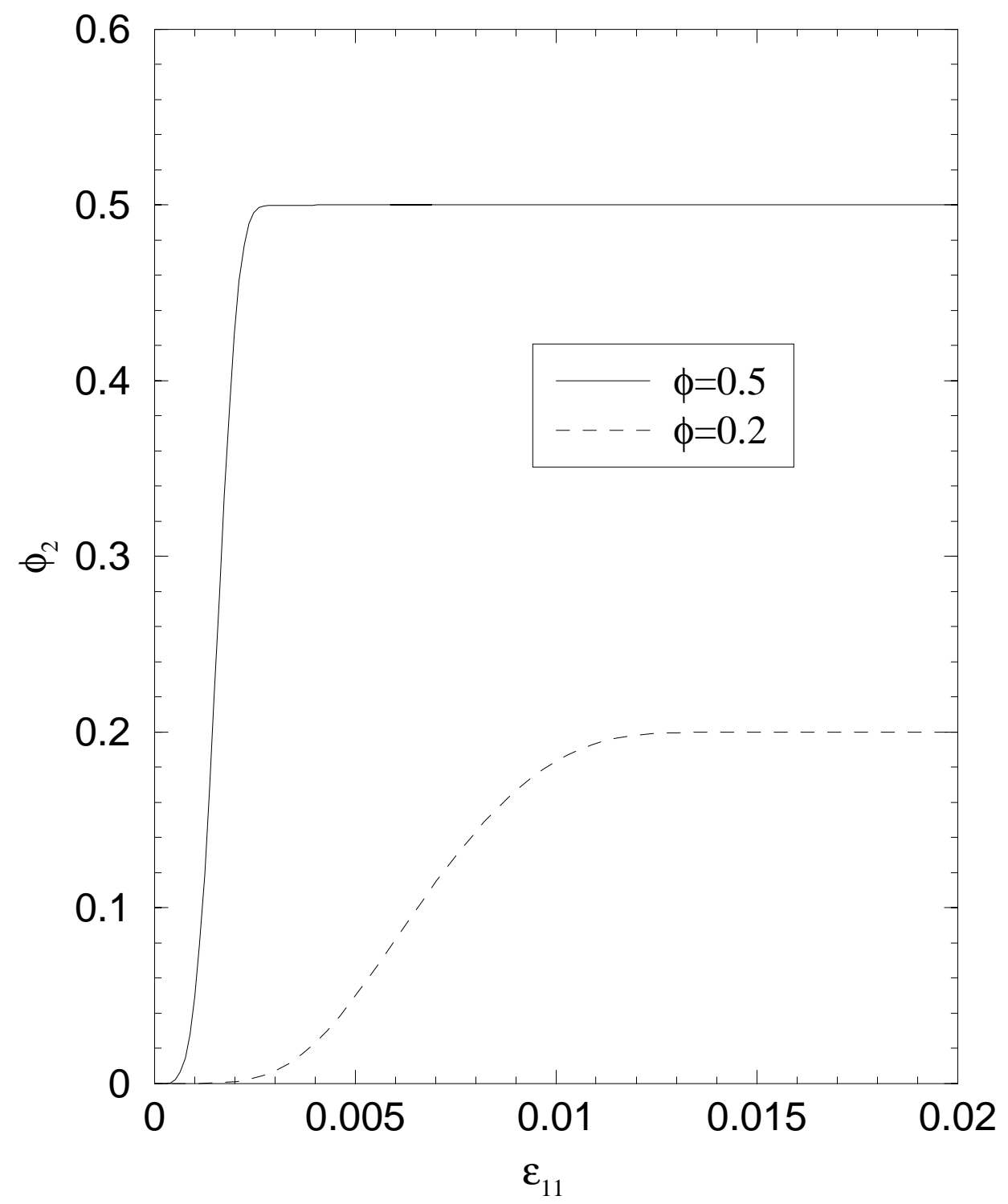

Figure 5. The predicted evolution of debonded fiber volume fraction corresponding to Figure 4. 


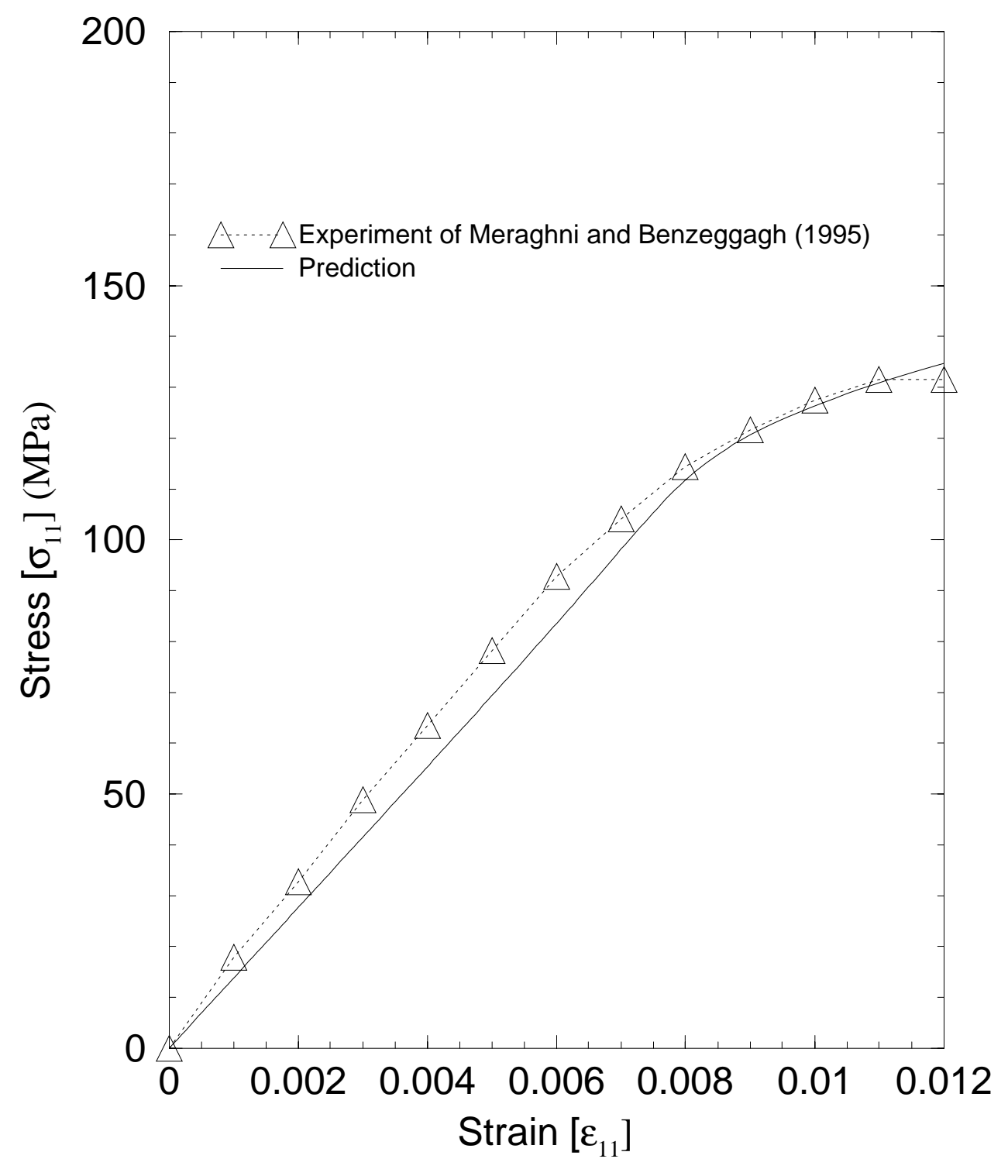

Figure 6. The prediction between the present prediction and experimental data (Meraghni and Benzeggagh, 1995) for overall uniaxial tensile responses of randomly oriented discontinuous fiber composites with initial fiber volume fraction of 0.5 . 


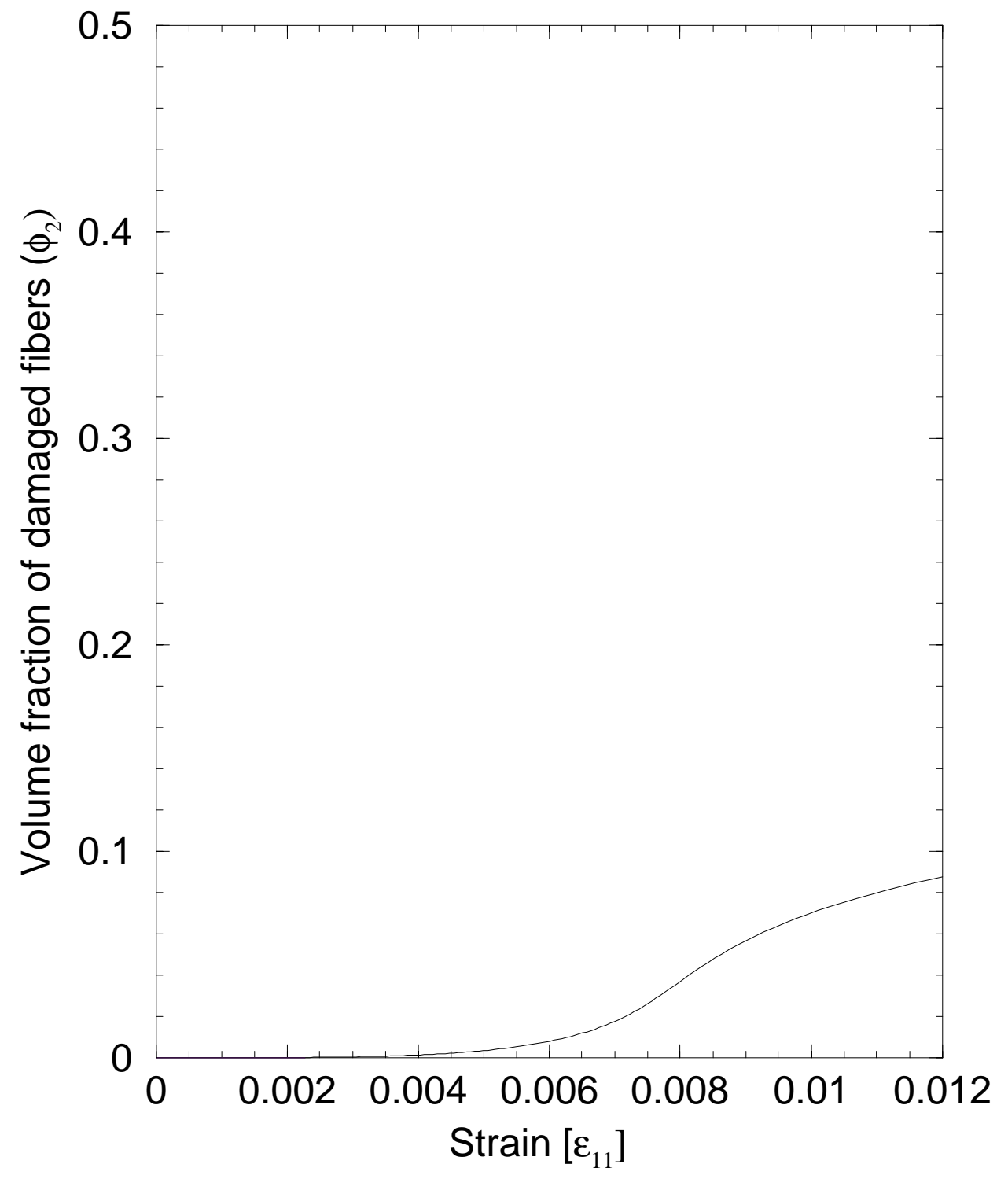

Figure 7. The predicted evolution of debonded fiber volume fraction versus strain corresponding to Figure 6. 
ORNL/TM-1999/158

\section{INTERNAL DISTRIBUTION}
1. P. Angelini
2. R. G. Boeman
3. R. A. Bradley
4. J. M. Corum
5. D. F. Craig
6. W. H. Gray
7. H. W. Hayden
8. P. Hughes
9. H. K. Lee
10. G. M. Ludka

11. B. Radhakrishnan

12. S. Simunovic

13. P. Sklad

14. M. J. Starbuck

15. D. Warren

16. T. Zacharia

17. R. Ziegler

18. ORNL Patent Section

19. ORNL Laboratory Records, RC

20. ORNL Laboratory Records (OSTI)

\section{EXTERNAL DISTRIBUTION}

21. DOE OFFICE OF SCIENTIFIC AND TECHNICAL INFORMATION, P.O. Box Q, Oak Ridge, TN 37831.

22. DOE, OFFICE OF HEAVY VEHICLE TECHNOLOGY, VEHICLE MATERIALS TECHNOLOGY PROGRAM, EE-RE, Forrestal Building, 1000 Independence Avenue, S.W., Washington, DC 20585

S. Diamond

23. DOE, OFFICE OF ADVANCED AUTOMOTIVE TECHNOLOGIES, LIGHTWEIGHT VEHICLE MATERIALS, EE-23, Forrestal Building, 1000 Independence Avenue, S.W., Washington, DC 20585-0121

J. A. Carpenter

24. DAIMLERCHRYSLER CORPORATION, 800 Chrysler Drive,

East Auburn Hills, MI 48326-2757

Sukhbir Bilkhu

25. DAIMLERCHRYSLER CORPORATION, CIMS 483-05-10, 800 Chrysler Drive, East Auburn Hills, MI 48326-2757

Venkatesh Agaram

26. FORD MOTOR COMPANY, Village Plaza, Suite 800, 23400 Michigan Avenue, Dearborn, MI 48124

Richard Jeryan

27. FORD MOTOR COMPANY, Village Plaza, Suite 800, 23400 Michigan Avenue, Dearborn, MI 48124

Hikmat F. Mahmood 
28. FORD MOTOR COMPANY, 20000 Rotunda Drive, P.O. Box 2053, SRL, MD3182 Rm. 2166, Dearborn, MI 48121-2053

Margaret Chadwick

29. FORD MOTOR COMPANY, 20000 Rotunda Drive, P.O. Box 2053, SRL, MD 2115 Rm. 2621, Dearborn, MI 48121-2053

Ari G. Caliskan

30. GENERAL MOTORS, 30500 Mound Road, P.O. Box 9055, Warren, MI 48090-9055 Mark E. Botkin

31. GENERAL MOTORS, 30500 Mound Road, P.O. Box 9055, Warren, MI 48090-9055 Nancy L. Johnson

32. GENERAL MOTORS, 30500 Mound Road, P.O. Box 9055, Warren, MI 48090-9055 Thomas J. Dearlove

33. LAWRENCE LIVERMORE NATIOAL LABORATORY, P.O. Box 808, L-126 Livermore, CA 94551

Ed Zywicz 